\title{
La profesionalización de la Filosofía en la Argentina a través de sus revistas Notas para la confección de un corpus hemerográfico
}

\section{Lucas Domínguez Rubio \\ Centro de Documentación e Investigación de la Cultura de Izquierda - CeDInCI I Universidad Nacional de San Martín - UNSAM I Consejo Nacional de Investigaciones Científicas y Técnicas - CONICET, Argentina / lucasImdr@autistici.org / https://orcid. org/0000-0001-9058-9573}

\section{Resumen}

El proceso de profesionalización académica de la filosofía se universalizó durante el siglo xx. Este proceso incluyó tanto una institucionalización de la disciplina como una problemática autonomización de la misma, que involucró de distintas maneras su secularización, su desvinculación de las esferas estatales, su separación del método científico de las ciencias duras, un paulatino alejamiento de otras áreas de las humanidades y su pretendida despolitización. Además el desarrollo de las humanidades dentro de las distintas unidades académicas requirió que la práctica filosófica adopte el formato de los artículos científicos. En el arco de estas modificaciones, este trabajo plantea ciertas hipótesis para identificar, clasificar y estudiar las revistas de filosofía realizadas en la Argentina a partir tanto de sus inscripciones académicas como político-culturales. En primer lugar, las páginas siguientes repasan las características de profesionalización propias de las revistas académicas, que estarían dadas por su modo de evaluación de los trabajos publicados, su especialización, su institucionalización y su función bibliométrica para la evaluación de investigadores. En un segundo momento, el trabajo propone una periodización de estas revistas desde la primera publicación universitaria con textos de filosofía durante los primeros años del siglo xx hasta las actuales revistas profesionales. En tercer lugar, este recorrido permite la identificación de distintas líneas teóricas de intervención filosófica, cultural y política. Por último, el presente desarrollo justifica la confección de una hemerografía anexa al trabajo que incluye las publicaciones periódicas argentinas sobre filosofía necesarias para el estudio de la profesionalización de la disciplina.

\footnotetext{
Abstract

The professionalisation of philosophy in Argentina through its journals: Notes for the preparation of a hemerographic corpus. The process of philosophy's academic professionalisation began in Europe during the 19th century and expanded worldwide along the 2oth century. Furthermore, the development of Human Sciences within academic spheres required the adaptation of that philosophical research in order to reach an academic magazine standard. This article aims to identify the aspects of the
}

\section{Palabras clave}

Publicaciones periódicas Pensamiento argentino y latinoamericano Procesos de profesionalización Filosofía Argentina
Keywords

Journals

Argentine and Latin American thought

Processes of professionalization Philosophy Argentina 
different philosophical Argentinean publications that are relevant for the study of the local professionalisation process, using academic, political and cultural issues. To begin with, we revise how this professionalisation has developed in academic publications in general. Secondly, we propose a timeline marking the periods of the publications, from the first university magazine to current professional journals. And thirdly, this study will enable the identification of different theoretical and political stances in these magazines and journals. Finally, in the Appendix, we create an index of journals and magazines including Argentinean publications about philosophy for the study of the discipline's professionalisation.

Artículo recibido: 2-10-2017. Aceptado: 2-04-2018

En cuanto a la revista, seguramente la situación política producida por el golpe de Onganía hará imposible la aparición del número próximo. También la universidad está afectada por el golpe de Estado. Una vez más, hay que empezar todo de nuevo.

\section{R. Piglia, Los diarios de Emilio Renzi}

Las primeras revistas con textos de índole científico surgieron en Europa a mediados del siglo xvii. Según Guedon (2001), respectivamente desde Paris y Londres, las revistas Journal des Savants (1665-1790) y Philosophical Transactions (1665-) cumplieron distintas funciones sociales, desde comunicar hipótesis y descubrimientos hasta constituir un registro de patentes durante el auge de la imprenta. Como señaló también este autor, se trataba de publicaciones previas a las divisiones disciplinarias que conocemos hoy en día y en general muchos de sus artículos pertenecían a lo que en ese momento se conocía como "filosofía natural".

Como sostiene Cassini (2017), el proceso de separación de la filosofía respecto a las ciencias naturales se asentó durante la segunda mitad del siglo xix. Sin lugar dudas, observar los distintos cambios dentro de las publicaciones periódicas de la disciplina permite identificar varias características de este proceso, en tanto hacia fines de este siglo ya existían revistas académicas especializadas en filosofía -entre ellas por ejemplo podemos nombrar Revue Philosophique de la France et de l'Étranger (Paris, 1876-), Mind (Oxford,1876-), FreiburgerZeitschrift für Philosophie und Theologie (Friburgo,1887-), Proceedings of the Aristotelian Society for the Systematic Study of Philosophy (Londres, 1888-), Archiv für Geschichte der Philosophie (Berlín, 1888-), Philosophisches Jahrbuch (Fulda, 1888-), The Monist (Illinois, 1890-1936; 1962-), International Journal of Ethics (Londres, 1890-), The Philosophical Review (Nueva York, 1892-), Revue de Métaphysique et de Morale (Paris, 1893-), Kant-Studien (Berlín, 1896) y Bulletin de la Sociétéfrançaise de philosophie (Paris, 1901-1990). Sin embargo, fuera de Europa y los Estados Unidos, el proceso de división disciplinaria se dio gradualmente recién durante el siglo xx con distintas características según cada lugar y sus tradiciones dominantes.

Con sus diferentes tiempos locales, este proceso de profesionalización involucró también una nueva relación entre los formatos de difusión de los textos filosóficos. Tradicionalmente la filosofía misma como disciplina ha privilegiado el análisis de libros. Por lo general se ha considerado que allí los distintos autores plasmaron las versiones definitivas de sus argumentos. Frente a esto, con el correr del siglo $\mathrm{xx}$, sin dudas la práctica filosófica de hoy en día ha tomado la forma de las revistas profesionales. Siguiendo el texto de Cassini (2017), si bien de ninguna manera este 
tipo de revistas reemplazó completamente al libro, esta transformación se instaló de manera más determinante en la filosofía anglosajona. En comparación, en la filosofía europea continental la importancia del libro es mayor y sus referentes más destacados prefieren aún hoy el formato monográfico. Aunque desde ya en ningún lugar los partícipes menos famosos del mundo académico pueden escapar de la exigencia de sus respectivas cuotas anuales de artículos.

Como veremos a continuación, al menos durante gran parte del siglo xx la producción filosófica local también apuntó siempre a llegar al libro. En muchos casos, una costumbre habitual de los profesores de filosofía de las primeras décadas del siglo consistió en publicar primeras versiones de sus trabajos en revistas culturales o universitarias, que de esta manera se transformaban en "laboratorios de ideas" y luego, solo en algunos casos, llegaban a publicarse, de un modo más acabado, en forma de libro. No obstante desde temprano los universitarios argentinos han dejado una buena cantidad de revistas enfocadas de distintas maneras hacia la filosofía. El objetivo principal de las siguientes páginas consiste en plantear ciertas hipótesis para identificar, clasificar y estudiar las revistas sobre filosofía aquí realizadas.

Con este fin, en primer lugar, se propone un corpus hemerográfico para responder a la pregunta ¿cuáles son las revistas a tener en cuenta para observar el desarrollo de la disciplina filosófica local? Se trata de un interrogante difícil. A diferencia de lo que sucede con otras áreas de conocimiento, los márgenes poco definidos de esta disciplina generaron que desde principios del siglo xx numerosas revistas hayan inscripto su agenda temática en diversas y hasta opuestas ideas sobre lo que es la filosofía. Frente a este problema, los criterios de selección para los objetivos del presente artículo han sido los siguientes. En general, la manera de responder esta pregunta ha sido seleccionar los medios en dónde publicaban los distintos actores académicos. De manera que el recorte queda a la vez constituido y reforzado al seguir las principales trayectorias personales bajo la pregunta ¿en dónde publicaron sus artículos, por ejemplo, Rodolfo Rivarola, José Ingenieros, Alejandro Korn, Coriolano Alberini, Francisco Romero, Carlos Astrada, Saúl Taborda o Luis Juan Guerrero? ${ }^{1}$ Este criterio también lleva a incluir revistas estudiantiles referidas a la actividad filosófica local. Si bien dentro de este conjunto algunas de las revistas incorporadas han publicado pocos textos sobre filosofía, todos los casos incluidos constaron de artículos e intervenciones que apuntaron a problematizar las prácticas de la disciplina misma. Como veremos, con este criterio incluimos revistas editadas por distintas universidades, facultades e institutos de filosofía. También bajo este criterio seleccionamos una importante cantidad de revistas culturales de amplio espectro, ya que, como señalaremos, al menos hasta la década del sesenta, estas constituyeron importantes plataformas donde distintos filósofos locales dieron a conocer una parte muy relevante de su producción. Por último, obviamente este criterio nos lleva a relevar las revistas profesionales de filosofía que se editan en el país desde al menos cuatro décadas.

En segundo lugar, desde ya el objetivo de estas páginas no consiste en brindar un análisis de todas las revistas relevadas, sino en presentar ciertas líneas de lectura. De esta manera el período histórico del cual se ocupa el artículo resulta sumamente amplio, ya comienza en los primeros años del siglo xx cuando no existían todavía revistas de filosofía propiamente dichas. Por un lado, esta perspectiva permite observar la continuidad de ciertos proyectos filosófico-culturales a través de las distintas revistas. Por otro lado, desde este punto de vista se pretende proponer una periodización de estas revistas en el proceso de desarrollo de la disciplina, para lo cual elegimos una clasificación retrospectiva de lo que hoy en día consideramos índices de profesionalización de una revista académica. De este modo, en ausencia de mayores trabajos al respecto y como parte de una investigación en curso, estas páginas proponen una cronología preliminar para ubicar las distintas revistas de filosofía publicadas en la Argentina.
1. Claro que principalmente se consumía filosofía editada fuera del país; sin embargo, este trabajo no trata sobre las revistas que se leían aquí sino sobre las que se editaron en la Argentina. Esta pregunta resulta mucho más difícil de responder para un momento donde ni los programas universitarios de las materias ni los artículos publicados se referían a la bibliografía que utilizaban de manera sistemática. Tampoco se conservaron muchos fondos personales y las autobiografías y memorias disponibles no especifican mucho al respecto. Aparentemente las únicas fuentes disponibles para este trabajo serían entonces las secciones de intercambios de las revistas y las referencias y citas realizadas en libros y artículos. 


\section{Las marcas de la profesionalización}

2. Aunque sí existen algunos acercamientos concretos. Biagini (1979 y 1985) trazó un mapa de las revistas y otros indicadores académicos de la actividad en la Argentina, como ser, entre otros, congresos, traducciones y reconocimientos en el extranjero. Gladys Palau (1995) analizó algunos aspectos de la revista Cuadernos de filosofía. Ramaglia (2010) propuso también ciertas hipótesis al respecto para pensar los cambios en la disciplina hacia 1918. Específicamente sobre las revistas de filosofía y su profesionalización seguimos de cerca el texto de Cassini sobre este tema publicado en 1998 y el trabajo de Romanos de Tiratel de 2008. Por el contrario, sí contamos con investigaciones sobre el proceso de profesionalización de las ciencias sociales (Altamirano y Sarlo, 2004; González, 2000)

la historia (Cattaruzza y Eujanian, 2003; Devoto y Pagano, 2009), la sociología (Blanco, 2006a y 2006b) y los estudios literarios (por ejemplo: Delgado, 2006).
Aunque es claro que cada revista adquirió características particulares, en las últimas décadas han aparecido un conjunto de reflexiones metodológicas sobre el tema que posibilitan identificar ciertos problemas propios de este tipo de documentos (Sarlo, 1992; Pluet-Despatin, 1999; Patiño, 2008; Delgado, 2014; Ehrlicher, 2014). Efectivamente en el último tiempo han proliferado tanto investigaciones sobre publicaciones periódicas como sobre la profesionalización de distintas áreas disciplinarias. Sin embargo, hasta ahora contamos con pocos estudios específicos sobre revistas de filosofía y sobre el proceso de institucionalización y academización de la filosofía en la Argentina².

Como veremos, las revistas filosóficas presentan ciertas características específicas que considerándolas en su serie se fueron modificando a lo largo de las décadas. $\mathrm{Al}$ respecto es importante remarcar que por las características propias de una disciplina fuertemente centrada en el análisis de los grandes libros, las revistas de filosofía no se constituyeron como objeto de estudio para los investigadores de la misma disciplina. La práctica filosófica misma prioriza la evaluación de argumentos y el análisis de conceptos y discusiones en buena medida abstractas. De manera que, al menos en Europa y América Latina, los libros siempre fueron considerados el lugar donde cada autor ha plasmado su pensamiento definitivo digno de ser analizado.

De este modo las historias de la filosofía en la Argentina con las que contamos hasta hoy en día (Farré, 1958; Alberini, 1966, 1981; Pro, 1973; Leocata, 1993 y Caturelli, 2001) principalmente se enfocaron en los textos publicados como libros, sin considerar de ninguna manera la intervención propuesta por una revista dentro del espacio cultural o académico. También los llamados estudios latinoamericanos han promovido principalmente investigaciones sobre ensayos, obras literarias, artísticas y políticas. Mientras también, como sostuvo Fernández Vega (2011), incluso desde los nuevos trabajos sobre historia intelectual en búsqueda de polémicas políticas de relevancia, los filósofos académicos argentinos por lo general han sido dejados de lado como objeto de estudio. Dentro de esta última línea, contamos sí con algunos estudios específicos sobre distintas revistas ligadas al ámbito filosófico local durante las primeras décadas del siglo xx (Rossi, 1999; Eujanian, 2001; Rodríguez y Vásquez, 2001; Bustelo, 2012, 2014; Prislei, 2015; Bustelo y Domínguez Rubio, 2016) y dos trabajos sobre las revistas profesionales de filosofía que seguimos de cerca (Cassini, 1998; Romanos de Tiratel, 2008).

Como indica su título, este trabajo buscará observar ciertos índices de profesionalización a través de las revistas de filosofía alrededor de los espacios académicos de la disciplina. Para ello, siguiendo el texto de Cassini (1998), partiremos de identificar ciertas características de lo que hoy en día consideramos una revista profesional en lo que respecta a sus modos de publicación, circulación y funcionamiento. ¿Cuáles serían estas marcas?

1. En primer lugar, un sistema de publicación mediante referato ciego que garantiza la evaluación de al menos dos especialistas en el área designados por un comité editorial. En alguna medida, este mecanismo evita que el director de la revista o su comité editorial seleccione cuáles artículos se publican. Desde ya este sistema no queda exento de críticas (entre otras, por ejemplo: Varki, 2017), pero es sin dudas el que funciona hoy en día.

2. En segundo término, la profesionalización involucra no solo un enfoque cada vez más centralizado en lo que se considera el campo filosófico, sino incluso la aparición de revistas dedicadas a áreas más específicas dentro de la filosofía, ya sea por tema, autores o una corriente de pensamiento en particular. De esta ma- 
nera, se proponen recortes temáticos que especifican aún más no solo su rango de intereses sino también su público lector, ahora conformado únicamente por especialistas en subtemas dentro de la misma disciplina.

3. En tercer lugar, en consonancia con los puntos anteriores, el hecho de que todos los textos de una revista sobre humanidades o ciencias sociales sean arbitrados convierte a la publicación en un banco de artículos, quitándole en gran medida la posibilidad de intentar otro tipo de intervención. La profesionalización conllevó una desaparición del espacio editorial, y únicamente las revistas terminan diferenciándose entre sí por su enfoque temático y sus características bibliométricas. Estas modificaciones involucran toda una serie de cambios en lo que se refiere a su vínculo con el lector y su forma de lectura y circulación. El lector-investigador se dirige a ella fundamentalmente en busca de artículos específicos sobre determinado tema y en casi ningún caso a la revista en su totalidad. Este fenómeno se acrecentó paulatinamente cuando las revistas digitales comenzaron a editarse digitalmente. A partir de este momento, los artículos útiles a cada investigador son rastreados por autor o tema mediante bases de datos científicas - como Scopus, Google Analytics o Scielo- mientras se busca expandir su visibilidad mediante redes sociales académicas - como ResearchGate y Academia. De manera que en buena medida las revistas son seleccionadas por sus autores según el beneficio que ofrecen por su trayectoria al posicionamiento del artículo. De este modo, claramente las revistas aumentaron su alcance; por ejemplo, las publicaciones científicas locales parecen poder ampliar sus límites nacionales de circulación a los que estaban confinados. Aunque, en tanto "banco de artículos", estas revistas perdieron también en buena medida sus respectivas características propias. Si bien, sin lugar a duda, esta es la tendencia general dentro de las revistas profesionales, recientemente algunas de ellas han intentado promover nuevas secciones con la publicación de algunos artículos no arbitrados o brindándole más espacio a las reseñas bibliográficas.

4. En cuarto lugar, todas las revistas profesionales poseen una inscripción institucional que las sostiene y un comité editorial de especialistas en el área con un comité asesor internacional. Fundamentalmente, esto le permite no sólo contactos profesionales para la evaluación de los artículos sino también herramientas de posicionamientos bibliométricos en las distintas bases de datos.

5. En relación con lo anterior, la aparición de distintos índices y bases de datos fundamentales para llevar a cabo cualquier investigación constituyen también una forma de evaluar cuantitativamente la producción de un determinado académico, en tanto la publicación en revistas prestigiosas se vuelve un hecho decisivo para obtener distintos tipos de puestos académicos. De manera que desde al menos cincuenta años son los libros $u$ otras revistas culturales donde los autores plasman hipótesis más atrevidas de intervención.

En síntesis, de manera general el proceso de profesionalización de la filosofía se universalizó durante el siglo xx. Este proceso incluyó tanto una institucionalización de la disciplina como una problemática autonomización de la misma, que involucró de distintas maneras su secularización, su desvinculación de las esferas estatales, su separación metodológica de las ciencias duras, un paulatino alejamiento de otras áreas de las humanidades y su pretendida despolitización. En cuanto a las revistas, en resumen, sus características de profesionalización estarían dadas por su modo de evaluación, su especialización, su confinamiento académico, su institucionalización y su función bibliométrica para la evaluación de investigadores. Desde ya que estos cambios en las publicaciones científicas manifiestan nuevas prácticas para los actores universitarios y estos indicadores de profesionalización de las revistas académicas no pueden tomarse aislados. No es el objetivo de estas páginas tratar de analizar el conjunto de estos cambios. Sin entrar en más detalles sobre los aspectos que involucra este proceso de profesionalización en cuanto a las revistas, nos interesa marcarlos 
3. Para este período he seguido el clásico texto de Gutiérrez (1868/1998) y los índices de estas publicaciones periódicas realizados por Maeder (1961, $1962,1965)$ y Auza $(1968,1980)$. como clara contraposición al ámbito y a las revistas desde las cuales partimos a principios del siglo xx argentino.

\section{Primeras revistas universitarias, estudiantiles y culturales}

En un primer momento, las revistas oficiales editadas por las distintas facultades dentro de la universidad en conjunción con algunas revistas culturales y científicas fueron el espacio de difusión de la producción de los profesores de filosofía locales: Anales de la Universidad de Buenos Aires (1896-1902), Revista de la Universidad de Buenos Aires (1902-1923; 1924-1931), Archivos de Psiquiatría y Criminología (1902-1913), Nosotros (1907-1943), Revista de la Universidad de Córdoba (1914-1972) y, más tarde, Humanidades (1921-1960). Un segundo conjunto estuvo dado por las revistas estudiantiles universitarias que publicaban, entre otras cosas, algunos artículos sobre filosofía. Este género se abrió con la publicación Filosofía y Letras (1901-1906), editada por egresados y estudiantes de la facultad. Al año siguiente de su cierre esta publicación fue continuada por el Boletín del Centro de Estudiantes (1907-1911) que en 1912 cambió su nombre por Verbum (1912-1936) y con el cual se mantuvo durante veinticinco años más.

Con anterioridad, la primera generación de profesores de la Facultad de Filosofía y Letras de la Universidad de Buenos Aires (UBA) - Rodolfo Rivarola (1857-1942), Juan Agustín García (1862-1923) y Ernesto Quesada (1858-1934), entre otros- habían participado de distintas maneras de las revistas culturales más importantes de las últimas décadas del siglo xix argentino - por ejemplo, de la Revista Argentina (1868-1872) de José Manuel Estrada, la Revista del Archivo General de Buenos Aires (1869-1872) de Manuel Ricardo Trelles (1821-1893), la Nueva Revista de Buenos Aires (1881-1885) de Vicente y Ernesto Quesada, o La Biblioteca (1896-1898) de Paul Groussac (1848-1929). ${ }^{3}$

Sin existir todavía un ámbito universitario enfocado en humanísticas, estas revistas del siglo xix argentino no publicaban artículos sobre ningún campo disciplinar dentro de lo que hoy en día consideraríamos dentro la filosofía. Como señaló Dotti (1992), durante el siglo xix la filosofía configuraba junto a la literatura y la historia un núcleo de intereses humanistas comunes. La conclusión negativa más simple es que no existieron publicaciones periódicas ni específicas ni medianamente referidas a la filosofía. Por el contrario, en su mayoría tanto los profesores de los colegios nacionales y la Facultad de Derecho como otros funcionarios de entidades estatales buscaron editar sus clases como texto monográfico en formato libro. De hecho, este fue el modo en que durante dicho período circularon los textos filosóficos, principalmente mediante manuales editados en Madrid, en Paris y, en menor medida, en Santiago de Chile y Lima. Sin considerar los textos sobre filosofía cristiana editados en Córdoba, desde el tomo de Rousseau publicado por Mariano Moreno en vísperas de la Revolución de Mayo hasta el año 1900, se habían editado en Argentina aproximadamente unos 45 libros sobre filosofía.

Algo parecido sucede al mirar las revistas consideradas científicas en aquellos momentos editadas desde espacios vinculados a las esferas estatales. Los Anales de la Sociedad Científica Argentina (1874-) probablemente fue la publicación más antigua de carácter profesional. No publicó sino algunos artículos referentes a digresiones de filosofía de la física realizados por autores de otros campos disciplinarios (Maeder, 1969). En cambio, ya entrado el siglo xx, dos revistas sobre derecho criminal y psiquiatría como Criminalogía Moderna (1898-1901) y su continuación Archivos de Psiquiatría y Criminología (1902-1913) -esta última editada por José Ingenieros (1877-1925) - publicaron textos ligados al enfoque biologicista que se impartía en la cátedra de psicología de la Facultad de Filosofía y Letras en esos años. Durante ese período aquí publicaron 
profesores como Coriolano Alberini (1866-1960), Daniel Cranwell (1870-1953), Carlos O. Bunge (1875-1918) y desde ya el mismo Ingenieros.

En este contexto, en 1904 se fundó la Revista de la Universidad de Buenos Aires (19021923; 1924-1931) en reemplazo de los Anales de la Universidad de Buenos Aires (1896-1902) que se habían dejado de publicar dos años antes y estuvieron principalmente orientados a la pedagogía universitaria. Esta nueva publicación del Consejo Superior de la Universidad fue puesta a cargo de Rodolfo Rivarola, en ese momento profesor titular en la Facultad de Filosofía y Letras. Durante su primera época, hasta 1923, escribieron allí también muchos de los profesores de esta Facultad, entre ellos: Carlos Octavio Bunge (en ese momento a cargo de la materia Ciencia de la Educación), Enrique Mouchet (1886-1977) (en la asignatura Lógica), José Nicolás Matienzo (18601936) (profesor titular de Lógica), Camilo Morel (1866-[1923]) (Estética), Horacio G. Piñero (1869-1918) (Psicología), Ernesto Quesada (Sociología), Alejandro Korn (18601936) (Historia de la Filosofía), Rodolfo Rivarola (Ética y Metafísica) y Coriolano Alberini (Introducción de filosofía). Los mismos profesores titulares, como parte de la Academia de esta Facultad, comenzaron en 1910 la edición de los Anales de la Academia de Filosofia y Letras (1910-1916) - presidida por Vicente Quesada hasta su fallecimiento-, los cuales, en definitiva, sirvieron para publicar libros completos de sus propios miembros ${ }^{4}$.

Con una propuesta de intervención similar, en los años siguientes se fundó la Revista de la Universidad de Córdoba (1914-1970) y más tarde Humanitas (1921-1960) de la Universidad Nacional de La Plata (UNLP), inicialmente dirigida por Enrique Mouchet (1886-1977), egresado de Filosofía y Letras de la UBA hacia 1910. Toda esta serie de revistas consistieron en publicaciones abiertas a todas las disciplinas universitarias, donde en un mismo número convivían artículos sobre pastos pampeanos, pedagogía y psicología biológica.

Por otra parte, si bien con anterioridad habían existido publicaciones estudiantiles dirigidas a la totalidad del aparato universitario, Filosofía y Letras (1901-1906) fue la primera revista editada por la primera camada de egresados y estudiantes de la Facultad homónima. Tras su desaparición, en 1907 se fundó el Boletín del Centro de Estudiantes de la Facultad de Filosofía y Letras de la UBA, del cual aparecieron diecinueve números hasta 1911. Al año siguiente cambió su título a Verbum (1912-1948) continuando con su numeración ${ }^{5}$. Estas revistas nacieron como banco de lecciones, apuntes y bolillas para los exámenes finales de las materias de la facultad a lo que sumaron textos de cátedra firmados por algunos profesores como Camilo Morel, Roberto Lehmann-Nitsche (1872-1938), Horacio Piñero, José Ingenieros y Alejandro Korn. Además cumplieron diversas funciones como avisar fechas importantes del calendario académico y se propusieron como un espacio de publicación para que los estudiantes de la facultad ensayaran sus primeros escritos. Al menos hasta 1908 no existió otro tipo de intervención estudiantil más que la de proveer textos para las materias ni hubo tampoco presencia de mayores disputas ${ }^{6}$.

Antes de crear en 1908 la revista Nosotros (1908-1943), los jóvenes Alfredo Bianchi (1882-1942) y Roberto Giusti (1887-1978) realizaban distintas tareas en este Boletín estudiantil como redactores. A diferencia de la inscripción universitaria de las revistas nombradas, Nosotros apuntó a un público más amplio con un mayor espacio para el desarrollo de estos temas. Aunque recién despuntaría en visibilidad durante la década siguiente, de esta manera Nosotros fue pionera en su género. Esta Revista mensual de letras, arte, historia, filosofía y ciencias sociales fue la primera en lograr un circuito culto común donde se sentían llamados a publicar hombres de todas las áreas y disciplinas humanísticas. Durante su tiempo de vida, muchos profesores universitarios de la facultad publicaron allí, entre otros: David Peña (1862-1930), Antonio
4. En cambio, desde 1902, ya existía la Revista de la Facultad de Derecho y Ciencias Sociales de la UBA específica de esta facultad, primero dirigida por Francisco J. Oliver y desde 1909 por Juan Agustín García, en ese momento profesor titular tanto en la Facultad de Derecho como en la Facultad de Filosofía y Letras. Durante sus épocas sucesivas esta revista se mantuvo como espacio donde publicaron también profesores de las siguientes generaciones como Lidia Peradotto, Alfredo Franceschi, Carlos Astrada, Ángel Vassallo, Luis Farré, Rafael Virasoro, Miguel Ángel Virasoro, Mario Bunge, Gregorio Klimovsky, Romero Brest, Rodolfo Mondolfo, Delfina Domínguez de Ghioldi, León Dujovne, Juan Carlos Torchia Estrada, Risieri Frondizi y Francisco Romero.

5. A partir de este momento se fundaron distintas publicaciones periódicas ligadas a diferentes institutos universitarios y facultades. El ciclo de revistas estudiantiles de la Facultad de Filosofía y Letras de la UBA puede ser recuperado gracias al trabajo de digitalización e indización realizado por el INIBI: http://inibi. institutos.filo.uba.ar/publicacion/ revistas-estudiantiles (INIBI, 2010).

6. Recién en el número siete del Boletín del Centro de Estudiantes apareció la voz de la Comisión Directiva al frente de una pequeña polémica. En una carta elevada al rector pedían revisar la designación de algunos profesores sustitutos que no consideraban capacitados para el cargo: Enrique del Valle Ibarlucea, Francisco De Veyga y Daniel Cranwell. A partir de ese momento también comenzaron a organizar algunas conferencias con invitados externos a la facultad como Saldías, Ameghino y Lugones. Al año siguiente, en 1908 este Centro de Estudiantes formó parte de la creación de la Federación Universitaria. Y desde estos primeros números del Boletín hasta el Boletín del Colegio de Graduados (1930-1944) puede rastrearse la lucha por el mayor reconocimiento de los títulos emitidos por la facultad en los concursos para profesores secundarios. 
7. Con todo, esta revista fue otra plataforma en la cual publicaron algunos de los profesores de la facultad como Camilo Morel, Horacio Piñero, José Nicolás Matienzo y Carlos Octavio Bunge. Sobre esta revista, ver: Roldán (2006).
Dellepiane (1864-1939), Alejandro Korn, Carlos Octavio Bunge, Francisco Romero (1891-1962), Carlos Astrada (1896-1970) y Luis Farré (1902-1997). Mientras además la revista se dedicó a publicar filósofos como Bergson, Brentano, Descartes y Dewey (Prislei, 2015). Como un emprendimiento en buena medida personal, pero dando lugar en sus páginas a los profesores de la Facultad de Derecho de Buenos Aires y La Plata, en 1910 Rodolfo Rivarola - profesor de la asignatura Ética y Metafísicadejó la dirección de la Revista de la Universidad de Buenos Aires y fundó su Revista Argentina de Ciencias Políticas (1910-1928), que promovió discusiones de derecho político y no contó más que con dos notas sobre áreas disciplinarias dentro de la filosofía $^{7}$. De esta manera durante este primer período convivieron revistas universitarias oficiales con algunas publicaciones estudiantiles dirigidas a la vida académica. De hecho, hasta 1917 es difícil encontrar artículos de estos profesores universitarios por fuera de estos medios. Y efectivamente también las primeras revistas universitarias y culturales fueron las plataformas donde Alberini, Astrada y Nimio de Anquín (18961979), entre otros, publicaron sus primeros textos. Aunque además durante los años siguientes esta primera camada de estudiantes dedicados principalmente a la filosofía participó de una nueva cantidad de medios impresos con características muy diferentes.

\section{Nuevas revistas en torno a la renovación universitaria}

La ampliación democrática se correspondió con un nuevo espacio público de debates y el ingreso de nuevos textos a las lecturas universitarias. Poco después esta apertura pareció exacerbarse con el entusiasmo local que despertó en muchos sectores la revolución rusa. Se trató entonces de una renovación política - caracterizada por un nuevo sentido democrático que permitía discusiones hasta ese momento inexistentes y se solapaba con diversos proyectos de izquierda en pugna-, social - signada por el aumento de las bases gremiales y el ingreso de las clases medias a la universidad-, cultural - con la aparición de un mercado para los libros y las revistas políticas, literarias y estudiantiles-, académica - con un desarrollo institucional más autónomoy también filosófica -en tanto a partir de allí se constató una importante apertura teórica a nuevos autores y corrientes.

Como demostró Natalia Bustelo (2014), las distintas publicaciones periódicas que dieron lugar a la reforma universitaria deben ser rastreadas a partir de 1914. Desde ya, estas revistas no deben ser consideradas revistas de filosofía, pero sí publicaciones de humanidades con textos cortos de intereses filosóficos que pretendieron una nueva interacción hacia dentro y fuera del ámbito universitario. De manera tal que con el correr de la década de 1910 los grupos estudiantiles buscaron politizar de distintas maneras este espacio en diálogo con otros actores de la sociedad. Así hacia 1918 coexistieron varios grupos con encontradas posiciones respecto a cómo debía ser la práctica filosófica universitaria.

En 1915 Ingenieros fundó la que habitualmente se considera la primera enfocada en la filosofía, precisamente la Revista de Filosofía, Cultura, Cienciasy Educación (1915-1929). A grandes rasgos, esta revista nucleó a quienes sostuvieron una idea de la filosofía de distintas maneras vinculada con las ciencias y buscó generar una continuidad cultural con la generación cientificista anterior (Biagini, 1985; Rossi, 1999). A diferencia de la mencionada revista de Rivarola que estaba en curso, las páginas editadas por Ingenieros proponían una agenda más dirigida a la filosofía europea y la historia cultural argentina. En contraposición al proyecto de Ingenieros y su revista, a partir de 1917 Alejandro Korn apoyó dos publicaciones estudiantiles anti-cientificistas con textos sobre filosofía y literatura: los Cuadernos del Colegio Novecentista (Buenos Aires, 1917-1921) y Atenea (La Plata, 1918-1919), dirigidas a intervenir en el ámbito académico 
de sus respectivas ciudades (Domínguez Rubio, 2017). Por su parte, Verbum, el ya nombrado órgano del Centro de Estudiantes, sufrió distintos cambios durante el transcurso de estos años. En líneas generales, el corte se dio durante 1919, cuando Gregorio Bermann (1894-1972), cercano a los círculos de Ingenieros, ya se había alejado de la dirección de la revista y, pocos números después fue asumida por Juan Probst (1892-1973), quien formaba parte de los cuadros novecentistas.

El Colegio Novecentista fue una institución estudiantil dedicada a la organización de conferencias, a la publicación de su revista y a la puja anti-positivista. Sus miembros se autoposicionaron como nuevos pensadores embanderados en las ideas del nuevo siglo en un ambiente que "bien poco sabía de las disciplinas filosóficas". Como el resto de las revistas nombradas, tuvo una sección de "Notas a la vida intelectual del mes", donde más que nada se evaluaban conferencias, libros y el accionar de algunas instituciones. En perspectiva, se diferenció de las revistas estudiantiles vigentes en esos años por la temática y la longitud de sus artículos, y se acercó al formato y modo de intervención que realizaban Nosotros y la revista de Ingenieros. De todas formas, tal como lo hizo Atenea, el intento fue trazar una disciplina filosófica mucho más cercana a la literatura, el arte y la cultura grecolatina, a lo cual Ingenieros y sus allegados se oponían (Eujanian, 2001). Según ellos mismos, rápidamente fueron acusados por sus supuestos "enemigos" de constituir una "facción católica". De manera que el Colegio Novecentista apareció como una primera publicación que prontamente derivó en posiciones antibolcheviques y elitistas que más tarde se convertiría en uno de los antecedentes de lo que Ingenieros caracterizó dos años después como "espiritualistas de derecha".

En 1918, Atenea: Letras - Artes - Filosofía irrumpió como una segunda revista con pretensiones de romper las posiciones teóricas hegemónicas. Vemos en su subtítulo que tampoco se trata de una revista especializada ni mucho menos filosófica; su irradiación temática se abría hacia otro lado, hacia la literatura y el arte. En su presentación y manifiesto, brindó un diagnóstico preciso que explicaba el ambiente en el cual la publicación pretendía marcar su novedad. Lo que presuponía un análisis de la historia ideológica argentina de las últimas décadas insinuado desde su manifiesto inaugural "Incipit vita nova". De modo que el claro rival frente al cual se posicionó Atenea también fueron el positivismo y el cientificismo como representantes del mecanicismo y el determinismo, y la novedad que quiso introducir consistía en una decidida preocupación ética (Bustelo y Domínguez Rubio, 2016).

Con todo, pese a su inscripción cultural fuertemente literaria, estas revistas iniciaron una sociabilidad de discusión filosófica que se inscribió en el conjunto de publicaciones más amplio propio de los primeros años de la reforma universitaria. En un gran arco de revistas estudiantiles, los posicionamientos teórico-políticos agruparon tanto a profesores como alumnos. Por un lado, existió un abanico de revistas estudiantiles que comenzaron a acercarse al socialismo (Bustelo, 2012, 2014). Por otro, hubo publicaciones que hasta 1921 buscaron inscribir la lucha estudiantil de la reforma universitaria en el ciclo social revolucionario que se abrió con la revolución rusa; entre ellas por ejemplo: Mente (Córdoba, 1920) de la que participaron Saúl Taborda (1885-1944) y Carlos Astrada; Cuasimodo (1919-1921) donde escribió Luís Juan Guerrero (1899-1957); e Insurrexit (1920-1921) de la cual participó Héctor Raurich (1903-1963) (Tarcus, 1997, 2004; Bustelo y Domínguez Rubio, 2017).

En todos los casos recién nombrados se trató de revistas-voceros de grupos vinculados a las universidades pero sin ninguna inscripción institucional oficial. Se trató de revistas vinculadas a un proyecto cultural, académico y hasta filosófico que buscaban ampliar la difusión de sus contenidos y el renombre de sus responsables. También al menos las revistas nombradas pretendieron darse a conocer como difusoras de 
un nuevo conjunto de textos, como evaluadoras de otros actores culturales y como pioneras de un nuevo modo de profesionalización, argumentando a favor de determinada concepción sobre lo que es la filosofía. A diferencia de las revistas universitarias, cada una de estas revistas filosóficas trazó una inscripción intelectual en relación con autores y revistas del exterior. Sin duda, las secciones de reseñas, debates y novedades editoriales conformaron un primer espacio de discusión ausente hasta ese momento. De modo que la capacidad de reseñarse y discutirse no dejaron de producir una naciente sociabilidad intelectual que conformó un campo disciplinario.

\section{La filosofía en el apogeo de las revistas culturales de la década del 20}

Como señalamos, al menos desde 1917, a través de revistas como Cuadernos del Colegio Novecentista o Atenea, los vínculos entre literatura y filosofía habían sido alentados como un nuevo espacio de exploración contra el cientificismo. Pero fue sin duda a partir de 1923 cuando fue claro que la práctica filosófica tal como la proponía Ingenieros había perdido totalmente sus posiciones. A partir de allí, una mayor cantidad de jóvenes lanzaron una nueva camada de revistas filosóficas-literarias enfocadas tanto en problemas teóricos como estéticos. Efectivamente, en 1923 se fundaron Inicial (Buenos Aires, 1923-1926), Valoraciones (La Plata, 1923-1928) y Córdoba (Córdoba, 1923-1927). Además de no estar dirigidas específicamente a la filosofía como disciplina, estas revistas no dejaron de mostrar diálogos con otras publicaciones específicamente literarias lanzadas durante esos años, como Proa (1922-1923), Martín Fierro (1924-1927), La Campana de Palo (1925-1927) y Clarin (1926-1927).

A partir de este momento quedaba claro que las generaciones más jóvenes de estudiantes y profesores de la disciplina abandonaban las revistas consideradas universitarias y científicas como únicos espacios donde publicar su producción. Veremos que, desde este momento y al menos hasta mediados del siglo xx, generalmente los actores de las carreras de filosofía han buscado al mismo tiempo publicar en revistas universitarias y culturales. Aunque lo que se destaca en este período es su acercamiento a las revistas específicamente estético-literarias. Saúl Taborda, Carlos Astrada, Ángel Vasallo (1902-1978), Miguel Ángel Virasoro (1900-1966) y Vicente Fatone (1903-1962) publicaron en los años siguientes tanto en las ya nombradas revistas universitarias como en las recién listadas revistas culturales, estudiantiles y estéticas (sobre alguno de estos casos ver: Velarde Cañazares, 2013). En cambio, por su parte, Coroliano Alberini fue uno de los primeros filósofos que desarrolló la totalidad de su producción en relación con revistas ligadas al ámbito universitario. También fue este autor quien a partir de 1927 comenzó a hacer explícita la necesidad de separar el discurso y la práctica filosófica de la literatura.

Durante la década del veinte el arco antipositivista quedó conformado de la siguiente manera. El elitista Colegio Novecentista opuesto a la desjerarquización universitaria y sus Cuadernos tuvo una deriva espiritualista religiosa, hasta el punto que la trayectoria de algunos de sus miembros puede ser rastreada en revistas que viraron al nacionalismo católico hacia fines de la década, lo que queda claro en el recorrido de Adolfo Korn Villafañe como mentor de los hermanos Irazusta o a la difusión de la filosofía escolástica dentro de la carrera de filosofía de la UBA por parte de Juan Probst. De manera que el proyecto reformista, axiológico y finalmente socialista de Alejandro Korn tuvo su plataforma de mayor ejecución en la revista Valoraciones (Bustelo y Domínguez Rubio, 2016). En ella se reunieron quienes asociaron la misión del estudiante y del filósofo a valores socialistas liberales y rivalizaron con otros grupos antiparlamentaristas, ya no ahora con aquellos entusiasmados con la revolución rusa sino más bien directamente con aquellos detrás del incipiente auge cultural de un nuevo nacionalismo autoritario. En esta línea, Valoraciones intercaló 
artículos sobre arte, filosofía e historia con manifiestos e ilustraciones que exaltaban a la juventud como guía moral con números especiales dedicados a Kant y Croce y referencias internacionales sobre Gandhi, Miguel Unamuno, José Ortega y Gasset, Eugenio D'Ors, Romain Rolland, Alfonso Reyes, Bertrand Russell y Waldo Frank. A fines de 1925, el joven que dirigía Valoraciones, Carlos Amaya, abandonó el proyecto para fundar junto a otros dos militantes reformistas, Carlos Sánchez Viamonte y Julio V. González, la revista Sagitario (1925-1927). Mientras Valoraciones quedaba a cargo de Korn, Sagitario sumaba a su antipositivismo un pronunciamiento antiimperialista vinculado a la Unión Latino-americana que Ingenieros había creado en 1925.

Como continuación de la nombrada revista anarco-bolchevique Mente (1920), en 1923 apareció Córdoba (1923-1925). Pese a los pocos números que se conservaron, podemos reconocer detrás de ella a los mismos jóvenes: entre ellos, a Taborda y Astrada. Respecto a su antecesora, y al igual que sus coetáneas en otras ciudades universitarias, esta revista reconoce el cierre del ciclo de entusiasmo revolucionario que se vivió en Europa y en los ámbitos obreros y estudiantiles de la Argentina. Con todo, la intervención politizadora que pretendió ejercer esta "revista de crítica universitaria", no renunciaba a propagar la discusión de textos de un vitalismo realista vinculado a lo social. Tras su cierre, Taborda y Astrada comenzaron a editar al año siguiente una revista mucho más enfocada al ámbito estético literario - Clarin (1926-1927) - que buscó principalmente una intervención cultural de quiebre vanguardista.

Mientras Cuadernos del Colegio Novecentista y después Valoraciones se centraron de alguna manera en la producción filosófica, la revista Córdoba se enfocó en la política universitaria. Por su parte, como señalamos, en un medio de consumos aledaños, la revista Inicial buscó dirigirse al espacio universitario, teórico y literario. Sin embargo, en este momento de especial interés del campo cultural por el vanguardismo y la estética, el caso de Inicial resultó especialmente significativo para las interpretaciones de los diferentes intereses teórico-políticos que se hicieron sobre el período. En sus páginas, el decadentismo de Spengler funcionó como un enlace en común a cierto irracionalismo estetizante y un continuo vitalismo nietzscheano que ya no aparecía leído ni junto a Sorel ni a Stirner ni tampoco en relación con ninguna otra referencia a la cultura de izquierda. Las interpretaciones clásicas que trazan una deriva directa entre el vitalismo irracionalista y el fascismo obtienen aquí entonces un importante cantero donde cosechar ejemplos. Dentro del arco de los filósofos, esta revista contó con el impulso de Homero Guglielmini (1903-1968), Miguel Ángel Virasoro y Vicente Fatone, mientras Astrada y Ángel Vassallo tuvieron breves colaboraciones en sus páginas. Con el correr de sus números, sin duda, las referencias al idealismo, al fascismo y al antijudaísmo de Inicial se volvieron fuertemente relevantes. Mientras centrándose en el primer editorial de la revista Francine Masiello (1986) propuso una interpretación de Inicial que enfatizó su patriotismo conservador para observar su posterior deriva, Fernando Rodríguez (2004) propuso una lectura completa de las tensiones dentro de la revista, queriendo dar cuenta de un "laboratorio de ideas donde nadie los conmina a hallar síntesis ideológica alguna"(43).

En síntesis, por un lado, durante la década del veinte, tenemos entonces revistas todavía apadrinadas por grandes figuras dentro del ámbito como Rivarola, Ingenieros y Korn. Aunque, por otro lado, en el recambio generacional de fuerte desprestigio teórico de buena parte de los "maestros", los "caudillos de prensa" filosóficos tendían a desaparecer. La gran mayoría de estas revistas culturales fueron producidas básicamente por estudiantes veinteañeros que, en mayor o menor grado, escudriñaban los vínculos entre literatura, filosofía y política. Teóricamente, buscaban distintas influencias y novedades para renovar el marco intelectual anti-cientificista mientras exploraban otros horizontes políticos que obtenían diferentes lecciones de la experiencia latinoamericana y rusa. Por ejemplo, en sus respectivas luchas con la tradición, 
como sostienen Rodríguez y Vásquez (2001), en La Plata la principal bandera no fue el anticlericalismo sino el antipositivismo. Mientras, en cambio, en el caso de Córdoba, el embate de los reformistas había tenido como uno de sus objetivos la lucha contra el dogmatismo clerical desde premisas liberales.

\section{Las primeras revistas institucionales de filosofía}

El proceso de profesionalización de la filosofía se asentó durante de la década del treinta con la creación de instituciones tanto públicas como privadas, cada una de las cuales erigió sus propias publicaciones periódicas.

Por fuera de la universidad, en 1930 se fundó la Sociedad de Estudios Kantianos encabezada por Korn-y el Colegio Libre de Estudios Superiores (CLES). También este último fue impulsado por Korn, en este caso junto a Roberto Giusti, Luis Reissig (1897-1972), Carlos Ibarguren (1877-1956) y Aníbal Ponce (1898-1938), quienes al año siguiente lanzaron su revista Cursos y Conferencias (1931-1952). En algunos puntos con un tipo de intervención similar a Nosotros, como su nombre lo indica, está revista principalmente publicó conferencias que se dictaron en el colegio sobre historia, geografía, sociología, derecho, política y filosofía. Así en los años sucesivos aparecieron textos de Francisco Romero, Astrada, Guerrero, Fatone, Risieri Frondizi (1910-1983), Manuel García Morente (1886-1942) y Eugenio Pucciarelli (1907-1995). Sin duda, a partir de 1943, el Colegio y su revista se mostraron como uno de los espacios de estudio por fuera de la universidad peronista en estrecha vinculación con distintos militantes del socialismo.

También en 1931 Victoria Ocampo fundó Sur (1931-1966). Aunque principalmente se trató de una revista con intereses literarios, rápidamente se convirtió en otra plataforma para las notas escritas por la terna de profesores de la facultad porteña. Entre otros, allí publicaron Astrada, F. Romero, Farré, Fatone, Pucciarelli, Guglielmini, Víctor Massuh (1924-2008) y Ezequiel de Olaso (1932-1996). Con el tiempo, la tarea editorial de esta revista resultaría fundamental. Como sucedió con el existencialismo sartreano, esta revista daría a conocer autores que no estaban presentes en los programas universitarios y que recién entrarían a los claustros a partir de la renovación universitaria iniciada en 1956.

Sin embargo, hasta este momento se trataba solo de revistas literarias y de humanidades que publicaban ocasionalmente algunos artículos de filosofía. La aparición de las primeras revistas exclusivamente con artículos sobre filosofía se dio paulatinamente dentro de instituciones universitarias y, en todos los casos, hasta la década del sesenta, estas resultaron esporádicas y discontinuas. En comparación a otras disciplinas este proceso parece haberse dado de manera algo tardía. Mientras, por ejemplo, el Instituto de Investigaciones Históricas de la Facultad de Filosofía y Letras de la UBA ya contaba con su propio Boletín desde 1922, los departamentos de filosofía de las distintas universidades tardaron al menos diez años más en tener sus propias revistas.

8. En el recambio generacional la camada anterior de profesores como Matienzo y Rivarola se alejaron paulatinamente de los claustros, pero siguieron publicando en revistas ligadas a la universidad como el Boletín del Colegio de Graduados de Filosofía y Letras (1930-1944).
Solamente a partir de 1934 se crearon las primeras publicaciones universitarias especializadas sobre filosofía. En Córdoba, el Boletín del Instituto de Filosofía de la Universidad Nacional de Córdoba (1934-1939) fue el primero en su género y tras su cierre fue continuado tres años después por Estudios de Filosofía en 1942. Dentro de la Universidad Nacional de La Plata, también en 1934 se creó el Centro de Estudios Filosóficos encabezado por Francisco Romero y su discípulo Eugenio Pucciarelli. Dos años después este instituto lanzó su publicación homónima Centro de Estudios Filosóficos (1936$1937)^{8}$. Pero esta revista no prosperó y recién en 1950 se creó su continuadora -la Revista de Filosofía - que sigue aún vigente. En la UBA, si bien en 1927 se había 
creado el Instituto de Filosofía, recién en 1948 durante la gestión de Carlos Astrada este tuvo su propia publicación, los Cuadernos de Filosofía (1948-1954; 1968-1978; 19891994; 1994-2004; 2008- ), que, tras varias etapas, cambios y reanudaciones, también sigue saliendo hoy en día.

En resumen, cada nueva universidad realizó según sus propios tiempos un ciclo similar en el cual precisaba sus publicaciones institucionales: primero de alcance universitario, después por facultad con la forma de revista de humanidades y finalmente por departamentos específicos. A pesar de su inscripción institucional, en un principio las revistas departamentales siguieron estrechamente unidas a las decisiones de su director, que en todos los casos fue el mismo director de departamento. Con todo, desde sus distintos proyectos editoriales, buscaban artículos de expertos y allegados, una revisión crítica de las obras editadas nacional e internacionalmente y un enfoque en los estudios filosóficos. Además, otra característica común, fue que las distintas revistas se presentaron desde un autoposicionamiento secundario respecto al marco filosófico internacional. Para ejemplificar estas características, tomamos el caso de la Revista de Historia de las Ideas creada en 1950 dentro de la Universidad Nacional de Tucumán y dirigida por Roger Labrousse (1908-1953):

Como es natural en una joven universidad que no posee todavía todos los elementos bibliográficos que serían necesarios para llevar a cabo investigaciones eruditas de un modo satisfactorio, los editores de la Revista de Historia de las Ideas no tienen la pretensión de ponerla a la altura de publicaciones editadas en los grandes centros de la cultura occidental. Al menos trataremos de aprovechar en la medida de nuestras fuerzas los recursos de los que disponemos y de conseguir la colaboración de estudiosos extranjeros que no tropiezan con los mismos obstáculos. Por otra parte, nos esforzaremos por imprimir a la revista de seriedad y austeridad, evitando no sólo los temas que por ser demasiado candentes y actuales no corresponden al historiador, sino también a toda concesión al deseo natural de ser agradables. (“Presentación”, Revista de Historia de las Ideas, $n^{\circ}{ }^{2}$ ).

En paralelo, las publicaciones filosóficas extra-institucionales exploraron otro tipo de intervención filosófico-política. Hacia mediados de la década del treinta nació un ciclo de medios tomistas y nacionalistas vinculados a la Unión Fascista Argentina, como Arx (1932-1935) y Sol y Luna (1938-1943). Si bien sus impulsores - respectivamente, Nimio de Anquín y Julio Meinvielle (1905-1973) - ya se desempañaban como profesores en universidades nacionales, esta línea logró inserción universitaria efectiva recién con la revista Arqué (1952-1956; 1962,1982) del Instituto de Metafísica e Historia de los Sistemas Metafísicos de la Universidad Nacional de Córdoba dirigida por Nimio de Anquín. Por su parte, en 1954 Julio Meinvielle -en la década siguiente "líder espiritual" del Movimiento Nacionalista Tacuara - creó Diálogo (1954-1955), que también nació y murió durante el peronismo, de modo que ambas revistas fueron clausuradas tras la llamada Revolución Libertadora.

Estas revistas mantenían publicidad cruzada con las publicaciones académicas de filosofía y teología que aparecieron también desde finales de la década del treinta desde la Universidad del Salvador y la Universidad Católica Argentina. En 1938 se fundó la revista Stromata y en 1944 se creó Ciencia y Fe (1944-1964). También, en la misma dirección, durante esos años la Universidad Católica Argentina lanzó su revista de filosofía Sapientia (1946-). Sin duda estas revistas generaron un ámbito de interacción y discusión propio. Solamente en muy pocos casos buscaron generar críticas y polémicas, sin lograr mayor eco en las publicaciones oficiales de las universidades nacionales. 
En estos años, desde sus puestos como profesores en la UNLP y la UBA, los discípulos de Alejandro Korn vinculados al socialismo - Francisco Romero, Luis Aznar, Sánchez Reulet, Ángel Vassallo y Eugenio Pucciarelli- organizaron una revista vinculada al proyecto de la unión democrática como Libertad Creadora (1943) (Bustelo y Domínguez Rubio, 2016). Con excepción de Vassallo y Pucciarelli, este grupo se desvinculó de los espacios universitarios durante el peronismo y se nucleó en la militancia cultural anti-peronista alrededor de Sur, Realidad (1947-1949) e Imago Mundi (1953-1956), para luego reingresar a la universidad tras la revolución libertadora y encabezar allí la renovación de los claustros.

También en esos años, surgió el proyecto de la revista Minerva (1944-1945) de Mario Bunge. Bunge lanzó esta publicación acompañado por Risieri Frondizi, quien recientemente había concluido sus estudios en la Universidad de Michigan. Se trató de una revista atípica para el momento dentro de esta serie de publicaciones. Emprendió desde fuera de la academia una militancia específica contra la línea fenomenológica que en aquel momento predominaba en las universidades de Buenos Aires y La Plata. En el mismo año de su cierre, fue la revista Episteme (1947-1971) la que, también desde fuera de la facultad, mantuvo la reflexión sobre filosofía de la ciencia. De manera que ambas revistas generaron un eslabón imprescindible anterior al ingreso del análisis filosófico en las facultades después de 1956. En este sentido, las distintas revistas de los institutos de filosofía obtuvieron cierta correspondencia con los núcleos temáticos que se dictaron en la facultad. Mientras por el contrario aquellas publicaciones aledañas fueron las que propusieron diálogos con teorías, textos o movimientos filosóficos fuera de los programas de estudio.

Con todo, según algunos testimonios, pese a la propagación de esta importante cantidad de títulos, aparentemente los artículos de estas revistas no tuvieron mayor injerencia en los programas universitarios. En buena medida porque, como señalamos, este no dejaba de ser un ambiente enfocado al análisis de libros. A lo que hay que sumarle que también eran espacios universitarios fuertemente dirigidos a la formación de docentes. Efectivamente, hasta la década del sesenta el egresado en filosofía no tenía una formación en investigación y, en todo caso, publicaba sus trabajos como texto monográfico por fuera de la universidad. Tras la llamada Revolución Libertadora, tampoco los institutos universitarios se mostraron como espacios estables para el desarrollo de investigadores sino que, por el contrario, resultaron especialmente permeables a los vaivenes políticos extra-académicos. Ambos motivos llevaron en la década del sesenta a la creación de instituciones privadas enfocadas en la investigación en filosofía.

\section{Las revistas profesionales de filosofía}

Si bien el Consejo Nacional de Investigaciones Científicas y Técnicas (CONICET) creado en 1958 había incorporado algunos investigadores en filosofía a partir de la década del sesenta, estos carecían de espacio de trabajo y bibliotecas especializadas. Como atestiguan algunas narraciones, debido la inestabilidad universitaria aparejada a los sobresaltos políticos, a partir de allí la actividad filosófica académica promovió la creación de nuevas instituciones privadas. Este problema de discontinuidad general se concretó en la llamada noche de los bastones largos. El desalojo de cinco universidades nacionales por parte del nuevo gobierno militar concluyó, según los casos, en la expulsión, renuncia y emigración de muchísimos profesores de todas las disciplinas: entre ellos, Risieri Frondizi, Gregorio Klimovsky (1922-2009), Tulio Halperín Donghi (1926-2014) y Sergio Bagú (1911-2002). Por ejemplo, según Eugenio Bulygin (1996), fue este el momento en que Carlos Alchourrón (1931-1996) propuso 
fundar una institución filosófica que fuera independiente de las injerencias oficiales y así nació la Sociedad Argentina de Análisis Filosófico (SADAF).

Bajo estas mismas motivaciones, en 1965 se fundó el Centro de Investigaciones Filosóficas (CIF) y seis años más tarde SADAF. Respectivamente, en 1975 y 1981 estas instituciones comenzaron a editar sus propias revistas académicas que todavía siguen en curso: la Revista Latinoamericana de Filosofía (RLF) y Análisis Filosófico. Como señaló Cassini (1998), la primera de estas revistas fue pionera en incorporar el doble referato ciego como medio de evaluación de los artículos, convirtiéndose así en la primera publicación profesional argentina de filosofía9. De manera discontinuada, paulatinamente, distintas revistas de filosofía también fueron impulsando este mismo mecanismo.

A partir de este momento y durante las décadas siguientes dentro de cada departamento de filosofía fueron apareciendo revistas específicas de cada área disciplinaria. En esta dirección, surgieron: Cuyo (1964-) dedicada a la historia de las ideas latinoamericanas; Ethos (1974-1975; 1995-1997) del Instituto de Filosofía Práctica (FFyL-UBA), reemplazada durante algunos números por Cuadernos de Ética (1986-); Patrística y Medievalia,(1975-) de la sección de estudios de Filosofía Medieval(FFyL-UBA); Orienteoccidente (1980-), revista de filosofía comparada editada por la Universidad del Salvador y el CONICET; la mencionada Análisis Filosófico (1981-) sobre filosofía analítica; Méthexis (1988-1997), revista de la Sección de Filosofía Antigua del Instituto de Filosofía de la UBA; e Hiparquía (1988-1999), sobre feminismo filosófico. Más recientemente aparecieron: Acontecimiento (1991-), publicación especializada en la producción de Alain Badiou y Louis Althusser; Perspectivas Nietzscheanas (1992-1998) e Instantes y Azares (2001-), dos revistas sobre las derivas de la filosofía de Nietzsche en el siglo xx; Cuadernos de Bioética (1996-); Prismas (1997-) y Políticas de la Memoria (1998-) sobre historia intelectual; y Deus Mortalis (2002-) dedicada a la filosofía política. También, por ejemplo, esta especialización paulatina se manifestó explícitamente en la revista Cuadernos del Sur editada por la Universidad Nacional del Sur de Bahía Blanca. Hasta 1992, publicaba un cuaderno anual interdisciplinario y a partir de esa fecha dividió sus distintas secciones de historia, letras y filosofía.

Claramente la cantidad de publicaciones periódicas sobre filosofía fueron en aumento y tomaron las características de profesionalidad que enumeramos en el primer apartado. En 1998 Cassini relevó la existencia de diez revistas argentinas registradas en Philosopher's Index. Diez años después, Romanos de Tiratel (2008) registró 37 revistas específicas de filosofía en curso, 17 editadas por instituciones públicas y 20 por institutos privados.

A modo de síntesis, primero, contamos entonces con la estabilización de publicaciones periódicas dirigidas a un público culto en general, como Nosotros, Sur, Punto de vista (1978-2008) y Espacios (1984-), las cuales se han mostrado como importantes plataformas de difusión de nuevas corrientes filosóficas a lo largo del siglo xx. En segundo lugar, revisamos revistas de humanidades dirigidas a un público universitario. Al principio estas estaban ligadas al estudio de otras disciplinas científicas y, más tarde, entre 1918 y 1927 principalmente poseían intereses estético-literarios. En tercer lugar, revistas a cargo de institutos o agrupaciones profesionales que apuntaron a un público especialista en filosofía. Y, por último, como sucede a nivel internacional, en cuarto lugar, nos referimos a la proliferación de publicaciones dirigidas al investigador especializado en áreas puntuales.

Este recuento concluye entonces con dos revistas de filosofía editadas por estudiantes y egresados que son críticas con este proceso de profesionalización académica. En primer lugar, la revista Dialéktica - realizada por profesores y estudiantes de las
9. Cassini (1998) subraya que el referato ciego es el índice fundamental que hace a una revista profesional. Contextualiza también en estos mismos años el auge de este tipo de revistas en otros países iberoamericanos: Dianoia (México, 1954-), Crítica (México, 1968), Diálogos (Puerto Rico, 1970-) y Manuscrito (Brasil, 1977-). Se trata también del momento en que nace la cienciometría destinada analizar cuantitativamente la producción científica a través de numerosas bases de datos. 
10. Por dar solo un ejemplo de esta crítica corriente desde algunas revistas estudiantiles de filosofía:

“a juzgar por las páginas de las revistas, [estas] escriben para sostener sus respectivos proyectos de investigación. Solo escriben para eso. Es decir, el horizonte de intervención de esos materiales parece limitarse a la auto-reproducción de los privilegios académicos. $\mathrm{Ni}$ siquiera parece que les interese dialogar con algún lector, ni dentro ni fuera de la Facultad de

Filosofía y Letras, salvando a sus pares, que se cuentan con los dedos de una mano y, ciertamente, a las agencias calificadoras de subsidios e incentivos". En:

“Editorial”, Amartillazos, n³, 2009,

p. 14 . Al respecto, ver también por ejemplo: "Editorial: máquinaestética”, Amartillazos, n 1, 2007. carreras de filosofía e historia de la UBA- publicó en su tercer número un análisis crítico retrospectivo sobre la participación con ponencias "filosóficamente abstractas" en el congreso de filosofía de 1980 del cual formó parte como invitado el presidente de facto Rafael Videla. Tomando este caso como hito de la autonomización disciplinaria, de esta manera, quince años después, el número 3/4 de Dialéktica abría de nuevo una pregunta latente a lo largo del proceso de profesionalización: ¿hasta qué punto como disciplina humanística la filosofía podía trazar su autonomía respecto a las otras esferas sociales?

En segundo lugar, otras publicaciones estudiantiles filosóficas también han criticado la escasa o nula circulación de las revistas académicas de filosofía. En este caso, su "hermetismo" es también entendido como una forma de inscripción a la escritura que exige un compromiso de despolitización respecto al lector ${ }^{10}$. Efectivamente, como sostiene Gladys Palau (1994: 42), ya no es posible encontrar referencias en las revistas de filosofía locales a la bomba atómica o incluso a la llamada noche de los bastones largos. Desde ya, esta es la característica propia de las revistas profesionales de todas las disciplinas que no han sido creadas para definirse en relación con elementos externos. Respecto a esto, algunos filósofos profesionales buscan otro tipo de plataformas para publicar intervenciones político-culturales y dirigirse a públicos más amplios con finalidades diferentes. E incluso es claro que aún hoy existen profesores e investigadores que han desarrollado su carrera exclusivamente a base de libros y publicaciones en revistas no profesionales. Sin embargo, es preciso señalar que se trata de un proceso en curso con una clara direccionalidad.

\section{Proyectos filosóficos y culturales: algunos lineamientos teórico- políticos a través de la producción de revistas de filosofía}

Como señalamos, alrededor de los espacios académicos, circularon revistas universitarias de los institutos de filosofía y también revistas políticas, culturales y literarias. Con el tiempo, estas publicaciones resultaron constitutivas de las actividades académicas imprescindibles del proceso de formación de un ámbito profesional, en lo que se refiere a las tareas de publicación, traducción y discusión. Las primeras revistas fueron lanzadas o apoyadas por importantes profesores del ámbito como Rodolfo Rivarola, José Ingenieros, Alejandro Korn, Francisco Romero y Coriolano Alberini, que ejercieron una función tutelar sobre sus distintas publicaciones. De allí en más, paulatinamente, fueron los consejos editoriales de los institutos universitarios los que editorializaban sus contenidos. En relación con las revistas profesionales de la actualidad, todas estas publicaciones plantearon cierta apertura desde la filosofía hacia la política cultural y universitaria. Por eso el bloque de revistas anterior al proceso de profesionalización de la prensa científica, que dentro de la filosofía comienza en 1975, no constituye un conjunto homogéneo. De manera que, en el correr de este trabajo, identificamos algunas de estas líneas de intervención político-cultural:

a) A partir de los índices de revistas vemos que entre 1918 y 1955 , en su gran mayoría, los artículos universitarios filosóficos se ocuparon de filosofía alemana y francesa. Especialmente predominaron textos sobre estética, axiología, bergsonismo, fenomenología y existencialismo heideggeriano. De manera correspondiente este abanico temático fue el que también, con distintas vertientes, se estableció en los programas universitarios. La línea cientificista social que abrió la Revista de Filosofía continuó con una publicación residual respecto a su contexto académico como fue El positivismo (1925-1938). Respecto al final del proyecto académico cientificista, como sostuvo desde su primer número la revista Minerva (1944-1945) - y como insinúa M. Bunge (2014) en su autobiografía - vino a llenar un espacio respecto a un ambiente filosófico copado por las derivas fenome- 
nológicas que reconocía a un antecedente lejano en la revista de Ingenieros. Por lo general, las notas sobre el ingreso sistemático de la filosofía anglosajona al campo de estudio local, destacan dos focos. Por un lado, mientras la revista Episteme (1947-1971) buscó configurar una filosofía de las ciencias por fuera de la FFyL, dos décadas después, el ingreso a esta facultad de Mario Bunge y Gregorio Klimovsky marcó una renovación de los programas universitarios. Por otro lado, fueron los grupos de estudio de la Facultad de Derecho de la UBA creados por Carlos Cossio (1903-1987) y Ambrosio Gioja (1912-1971) los que también contribuyeron a la estabilización de estudios sobre filosofía analítica que una década después dieron lugar a SADAF y su revista Análisis Filosófico (1981-); sin duda una de las instituciones de producción teórica partícipe de la transición democrática (Rabossi, 1981; Maffia, 2010). Desde ya, si bien Bunge y Minerva le hicieron un reconocimiento a la filosofía científica de Ingenieros como su antecedente, los dos textos recién nombrados a cargo de Rabossi y Maffia recapitularon el ingreso de la filosofía analítica al país sin remontarse hasta lejos.

b) A través de las trayectorias de Saúl Taborda, Carlos Astrada y Luis Juan Guerrero, puede trazarse un recorrido político similar, que fue de la militancia anarquista durante los primeros años de la década del veinte a la adscripción a FANOE (Frente de Afirmación del Nuevo Orden Espiritual) y el nacionalismo de la década del treinta. En buena medida, este recorrido común queda materializado a través de las revistas Mente (1920), Córdoba (1923-1925), Clarín (1926-1927) y Facundo (1935-1939). Como continuación, en el caso de Astrada y Guerrero durante las dos décadas siguientes ambos dirigieron el Instituto de Filosofía durante la universidad peronista y fueron expulsados de sus cargos, respectivamente, en 1955 y 1956.

c) Al menos desde Arx (1933-1934), Sol y Luna (1938-1943) y Diálogo (1954-1955) de Julio Meinveille se abrió una línea de revistas nacionalistas-tomistas cercanas a grupos fascistas locales y una inserción académica concreta en la Universidad Nacional de Córdoba con la revista Arkhé (1952-1955; 1962; 1982). Si bien los vínculos ideológicos entre estas revistas no siempre fueron explícitos, es posible conectarlas al unir trayectorias personales y observar las publicidades cruzadas entre ellas. Llamativamente, a través de Alberto Buela (1946-), discípulo declarado de Nimio de Anquín, esta línea dentro del pensamiento local tiene su continuación actual en formato YouTube en el canal nacionalista de derecha Toda la verdad primero (TLV1) del Partido Segunda República (PSR).

d) Con la figura de Alejandro Korn como bandera, el proyecto socialista-liberal iniciado en Valoraciones fue continuado por instituciones extra-académicas que resultaron fundamentales para el proceso de profesionalización de la filosofía local. La revista Cursos y conferencias sirvió como un importante medio de difusión de la producción de muchos profesores. Además, otra revista como Libertad creadora (1943) realizada por discípulos de Korn durante la experiencia de la Unión Democrática buscó resignificar luego también su legado, como también lo hizo desde fuera de la universidad Francisco Romero con la revista Realidad (1947-1949).

e) Debido a la importancia que tomó la obra de José Gaos (1900-1969), Leopoldo Zea (1912-2004) y Arturo Ardao (1912-2003), en 1964 una reunión de decanos de las universidades nacionales recomendó incluir el estudio del pensamiento argentino en los curricula académicos. Con Diego F. Pro (1915-2000) a la cabeza, la Universidad de Cuyo creó una revista específica sobre el tema Cuyo (1964- ), donde, entre otros, Arturo Roig (1922-2012) y Enrique Dussel (1934-) publicaron sus primeros textos. Con posterioridad, estos autores, a contramano de la de las inclinaciones políticas de su mentor, desarrollaron distintos proyectos intelectuales orientados a una filosofía latinoamericana con posicionamientos levinasianos y descolonizadores. Estos proyectos se plasmaron en varios libros y al menos en dos publicaciones periódicas: la Revista de Filosofía 
Latinoamericana (1975-1979; 1985-2000) y Estudios (2000-2004), esta última llevada a cabo por Roig. Esta línea continúa de distintas maneras en el proyecto de historizar el pensamiento alternativo por parte de Hugo Biagini y, dentro de la UBA, en los textos latinoamericanistas de Carlos Cullen y Alcira Bonilla.

En resumen, si bien es posible trazar estas líneas político-culturales, por lo general predominan numerosas revisiones genealógicas con formas de homenaje y, excepto algunos pocos casos, no existen discusiones con la generación anterior de filósofos locales. Por el contrario, las revistas muestran artículos de análisis de pensadores principalmente europeos y, solo desde las últimas cuatro décadas, también anglosajones. Lo que - solo con excepciones- no generó diálogos sino un reconocido sentido unidireccional del conocimiento. Por esto, como tendencia general pero especialmente desde las revistas profesionales de las últimas décadas, en comparación con las publicaciones del exterior, sus páginas quedan exentas de polémicas.

En relación a esto, como señalamos, durante las últimas décadas del siglo xx, la filosofía académica se emancipó del formato monográfico del libro. Sin duda no sólo fue el momento en que la filosofía se academizó definitivamente, sino también el momento en el que se materializó en formato revista. Con todo, también, en este caso se trata de un proceso en curso. Tal como lo hizo Sarlo (1992), Gladys Palau (1994) sostuvo que las revistas de filosofía también hasta principios de los años noventa constituyeron un "laboratorio de experimentación", pero, en este caso, todavía como artículos de destreza en el manejo de un tema que se presentaba como pasos previos al libro. Por el contrario, hoy en día el propio sistema de evaluación de las principales instituciones académicas desalienta esta clase de publicación.

\section{Agradecimientos}

Agradezco los esmerados comentarios de los evaluadores anónimos de este artículo así como el espacio de trabajo con las publicaciones periódicas generado en el CeDInCI. 


\section{Anexo: Hemerografía filosófica argentina ${ }^{11}$}

1 ACONTECIMIENTO. Revista para pensar la política. Buenos Aires: Grupo Acontecimiento, no 1: julio 1991 - [publicación abierta]. Director Raúl Cerdeiras.

2 AGORA PHILOSOPHICA. Revista Marplatense de Filosofía. Mar del Plata: Asociación Argentina de Investigaciones Éticas, $n^{\circ}$ 1: 2000 - [publicación abierta]. Comité editorial inicial: Graciela Fernández, Leandro Paolicchi, Romina Pulley, Romina Conti, Gustavo Salerno, Diego Parente, Andrés Crelier, Leandro Catoggio, Ricardo Maliandi, Herminia Solari, Luis Varela.

3 ALETHEIA. filosofía, ciencia, literatura, arte. Buenos Aires: [s.n.], 1956-1957. Director: Eugenio C. Zerba. Secretario de Redacción: Osvaldo D. Romano.

4 AMARTILLAZOS. revista de filosofía, estética y política. Buenos Aires: Colectivo de Trabajo Amartillazos, $n^{\circ} 1$ (otoño 2007) - [publicación abierta].

5 AMITICIA. revista de universitarios de filosofía y letras. Buenos Aires: Centro Universitarios de Acción Católica, $n^{\circ}$ 1: 1941 - n $39: 1954$.

6 ANALES DE LA ACADEMIA DE FILOSOFÍA Y LETRAS. Buenos Aires: FFyL-UBA-Academia de Filosofía y Letras, $n^{\circ}$ 1: $1910-n^{\circ} 6: 1916$.

7 ANALES DE LA UNIVERSIDAD DE BUENOS AIRES. Buenos Aires: FFyL-UBA, 1888-1902. Continúa en 1902 como Revista de la Universidad de Buenos Aires $\rightarrow$.

8 ANÁLISIS FILOSÓFICO. Buenos Aires: Sociedad Argentina de Análisis Filosófico, $\mathrm{n}^{\circ}$ 1: 1981[publicación abierta]. Consejo de dirección inicial: Eugenio Bulygin, Carlos Nino, Raúl Orayen, Eduardo Rabossi, Félix Schuster. Secretaría de redacción inicial: María Cristina González, Cecilia Hidalgo, Diana Maffia, Gladys Palau.

9 ANUARIO BIBLIOGRÁFICO. Letras, historia, educación y filosofía. La Plata: UNLP-FaHCEInstituto Bibliográfico, 1927-1929.

10 ANUARIO DE FILOSOFíA. Tucumán: Facultad de Filosofía y Letras - Universidad Nacional de Tucumán, nº1: 1982-.
11 ANUARIO DE FILOSOFÍA JURÍDICA Y SOCIAL. Buenos Aires: Asociación Argentina de Derecho Comparado, 1983-199o. Dir.: Martín Laclau.

12 ARQUÉ. Revista de metafísica. Córdoba: UNCInstituto de Metafísica e Historia de los Sistemas Metafísicos, $n^{\circ} 1$ : octubre $1952-1982$. Dir.: Nimio de Anquín. Co-director: Belisario Tello.

13 ARX. Córdoba: Instituto Santo Tomás de Aquino, $n^{\circ} 1$ : $1933-n^{\circ} 3$ : 1934. Dir.: Nimio de Anquín.

14 ATENEA. Letras - Artes - Filosofía. La Plata: Asociación de ex-alumnos del Colegio Nacional, no 1: 1918 - no 12: 1919. Director: Rafael Alberto Arrieta. Secretario de redacción: Luís $\mathrm{H}$. Sommariva.

15 AVATARES FILOSÓFICOS. Revista del Departamento de Filosofía. Buenos Aires: UBA-FFyL-Departamento de Filosofía, $n^{\circ}$ 1: 2013[publicación abierta]. Dir.: Claudia Mársico. Secretarios de redacción: Ezequiel Pinacchio, Matías Soich. Comité editor: Alcira Bonilla, Claudia Jáuregui, Pamela Abellón, Karina Pedace, Ailín Flaminman, Manuel Tangorra.

16 BASES. Buenos Aires: FFyL-UBA-Centro de Estudiantes, $\mathrm{n}^{\circ}$ 1/2: 1940.

17 BIBLIOGRAFÍA ARGENTINA DE FILOSOFÍA. La Plata: Ministerio de Educación de la Provincia de Buenos Aires-Instituto de Bibliografía, 1960-1969.

18 BOLETíN DEL CENTRO DE ESTUDIANTES DE FILOSOFÍA Y LETRAS. Buenos Aires: UBA-CeFyL, 1907-1911. A partir de 1912 continúa como Revista del Centro de Estudiantes de Filosofía y Letras y luego como Verbum $\rightarrow$.

19 BOLETíN DEL COLEGIO DE GRADUADOS DE LA FACULTAD DE FILOSOFÍA Y LETRAS. Buenos Aires: Colegio de Graduados de la Facultad de Filosofía y Letras, $n^{\circ} 1$ : octubre $1930-n^{\circ} 33 / 34$ : 1944.

20 BOLETíN DE EPISTEMOLOGÍA. Buenos Aires - La Plata: Sociedad Argentina de Filosofía, 19501951.

21 BOLETíN DEL INSTITUTO DE FILOSOFíA. Córdoba-Instituto de Filosofía: UNC, ${ }^{\circ}{ }^{\circ}$ : $1934-$ $\mathrm{n}^{\circ}$ 22: 1939.

11. La presente hemerografía consigna alfabéticamente las publicaciones periódicas seleccionadas bajo los criterios desarrollados. Cada entrada específica: título, subtítulo, lugar de edición, editor y fechas límite. A continuación se nombra el director o comité editorial de la revista según constó en el primer número de cada una. La información que se presenta de manera conjetural queda especificada entre corchetes "[---]". La flecha “ $\rightarrow$ " indica una referencia interna dentro de esta misma hemerografía. 
22 BOLETÍN INFORMATIVO FILOSOFÍA. Buenos Aires: Fundación para el Estudio del Pensamiento Argentino e Iberoamericano, [ $\mathrm{n}^{\circ}$ 1: 1984-].

23 CÁTEDRA DE INTRODUCCIÓN A LA FILOSOFíA. Buenos Aires: UBA-FFyL-Departamento de Filosofía, [1965-1967].

24 CENTRO. Revista del Centro de Estudiantes de Filosofía y Letras de la Universidad de Buenos Aires. Buenos Aires: CeFyL, no 1: noviembre 1951 no 14: cuarto trimestre 1959. Comité de dirección: Darío Cantón, Noé Jitrik, Ana Goutman, Esther Smud, Adelaida Gigli, Adolfo Prieto, Ismael Viñas, Ana llstein, Rodolfo Borello, etc. Eliseo Verón dirigió los no 11 y 12; Jorge Raúl Lafforgue los no 13 y 14 .

25 CENTRO DE ESTUDIOS FILOSÓFICOS. La Plata: UNLP, 1936-1937. Dir.: Eugenio Pucciarelli.

26 CIENCIA Y FE. San Miguel (Pcia. de Buenos Aires) - Buenos Aires: Universidad del SalvadorFacultad de Filosofía y Teología, $\mathrm{n}^{\circ}$ 1: $1944-\mathrm{n}^{\circ}$ 20: 1964. Dir.: Jaime Amadeo.

27 CLARín. Revista de crítica y cultura. Córdoba: [s.n.], $n^{\circ} 1$ : 30/8/1926 - n ${ }^{\circ}$ 12/13: 13/6/1927. Dir.: Carlos Astrada, luego Saúl Taborda.

28 CóRDOBA. Decenario de Crítica Social y Universitaria. Córdoba: [s.n.], № 1: 1923 - [nº 69: julio 1925].

29 CORREO DE CEFyL. Publicación del Centro de Estudiantes de Filosofía y Letras. Buenos Aires: CeFyL, no 1 (junio 1962) - no 3 (1962).

30 CUADERNOS DE BIOÉTICA. Buenos Aires: AdHoc, nº: 1996 - [publicación abierta].

31 CUADERNOS del Colegio Novecentista. Buenos Aires: Colegio Novecentista, $\mathrm{n}^{\circ}$ 1: julio 1917 - $\mathrm{n}^{\circ}$ 10: 1921. Dir.: Santiago Biggi.

32 CUADERNOS DE ÉTICA. Buenos Aires: UBAFFyL-Centro de Investigaciones Éticas "Dr. Risieri Frondizi”, № 1: 1986 - [publicación abierta]. Dir.: Ricardo Maliandi.

33 CUADERNOS DE FILOSOFÍA. Buenos Aires: UBAFFyL-Instituto de Filosofía, [primera época:] $\mathrm{n}^{\circ}$ 1: 1948 - no 13/14/15: noviembre 1953/diciembre 1954 (Dir.: Carlos Astrada); segunda época: $n^{\circ} 1: 1968$ - no 28/29: 1978 (Dir.: Eugenio Pucciarelli); Tercera época: $n^{\circ}$ 30: 1989- n³9: 1994 (Comité de redacción: Mario Caimi, Margarita Costa, Alberto Moretti, María Isabel Santa Cruz); quinta época: $n^{\circ}$ 40: 1994 - nº 49: 2004 (Comité de redacción: Gregorio Kaminsky, Carlos Alchourrón, Margarita Costa, Oscar del Barco, Jorge Dotti, Jorge Lovisolo, Arturo Roig y José Sazbón); [sexta época]: $n^{\circ}$ 50: 2008 - [publicación abierta].

34 CUADERNOS DE FILOSOFÍA ANTIGUA. Buenos Aires: Biblos, $n^{\circ}$ 1: 1979- [nº 8: 1985].
35 CUADERNOS DE FILOSOFÍA Y POLÍTICA. Rosario: Universidad Nacional de RosarioFacultad de Humanidades y Artes-Escuela de Filosofía, ํㅜ 1: 2000 -.

36 CUADERNOS DEL SUR. Bahía Blanca: Universidad Nacional del Sur- Departamento de Humanidades, $\mathrm{n}^{\circ}$ 1: 1958 - [publicación abierta]. A partir del $n^{\circ} 25$ (1992) la publicación se divide en tres secciones, una de ellas específica sobre filosofía.

37 CUADERNOS FILOSÓFICOS del Instituto de Filosofía. Rosario: Universidad Nacional del Litoral, [primera época:] n 1 (1960) - nº 4 (1963); segunda época: 1969-1970; Tercera época: $n^{\circ} 1$ : 2004 - [publicación abierta]. Primeros directores: Adolfo Carpio y Ángel J. Cappelletti.

38 CUESTIONES DE FILOSOFíA. Buenos Aires [s.n.], no 1: primer trimestre $1962-n^{\circ}$ 2/3: segundo/ tercer trimestre 1962. Dir.: Marco Aurelio Galmarini, J. Arthur Giannotti, Jorge Lafforgue, León Sigal, Eliseo Verón.

39 CURSOS Y CONFERENCIAS. Revista del Colegio Libre de Estudios Superiores. Buenos Aires: CLES, no 1 (julio 1931) - no 288 (julio/diciembre 1960).

40 CUYO. Anuario de filosofía argentina y americana. Mendoza: Universidad Nacional de Cuyo- Facultad de Filosofía y Letras-Instituto de Filosofía Argentina y Americana, $\mathrm{n}^{\circ}$ 1: 1964 [publicación abierta]. Primer director: Diego Pro.

41 DEBATES Y COMBATES. Buenos Aires: Asociación Civil Escenarios, $n^{\circ} 1$ (noviembre 2011) - no 6 (2014). Dir.: Ernesto Laclau.

42 DEUS MORTALIS. Cuaderno de filosofía política Buenos Aires: UBA-FFyL-Centro de Estudios de Filosofía Política y Social, $n^{\circ}$ 1: 2002- [publicación abierta].

43 DIALÉKTICA. Revista de filosofía y teoría social. Buenos Aires: Secretaría de Asuntos Académicos Estudiantiles CEFYL, nº 1: junio 1992-[publicación abierta]. Consejo editor inicial: Alfredi Bonnet, Darío Díaz, Gustavo Fondevila, Néstor Kohan, Sebastián abad, Patricio Enrique Mc Cabe, Eduardo Emilio Glavich, Florencio Noceti, Mariano Repossi, Verónica Lía Zalochi, Marcelo Dimenstein.

44 DIÁLOGO. Revista trimestral. Buenos Aires: Colegio de Estudios Universitarios de Buenos Aires, $n^{\circ} 1$ (primavera 1954) - ${ }^{3} 3$ (otoño/invierno 1955). Dir.: Julio Meinvielle.

45 DIAPORIAS. Revista de filosofía y ciencias sociales. Buenos Aires: UBA-FSOC, $n^{\circ}$ 1: noviembre 2002 - [publicación abierta]. Dir.: Rubén Dri. 
46 DIÓGENES. Revista internacional de ciencias humanas publicada con los auspicios del Consejo Internacional de Filosofía y Ciencias Humanas y con el concurso de UNESCO. Buenos Aires: Sudamericana, no 1 (octubre 1952) - no 96 (1976). Comité directivo: Denis W. Brogan, Alfonso Caso, Daya Krishna, Gilberto Freyre, Francesco Gabrieli, Max Horkheimer, Richard P. McKeon. Jefe de redacción: Roger Caillois. .

47 DISCURSO Y REALIDAD. San Miguel de Tucumán: [s.n.], ํo 1: septiembre 1985 - 1994. Dir.: Rolo Maris. Asesores: Hernán Zucchi, Hernán y Roberto Rojo.

48 DISENSO. Revista de metapolítica. Buenos Aires: Fundación Cultura et Labor, ํㅜ 1 (1994) - no 16 (invierno 1998). Dir.: Alberto Buela.

49 EIDOS. Revista de filosofía. Córdoba: UNCFacultad de Filosofía y Humanidades, $n^{\circ}$ 1: 1969 - n 3: 1972. Dir.: Alberto Caturelli. Continúa a Xenium $\rightarrow$.

50 ENSAYOS Y ESTUDIOS. Revista de filosofía y cultura. San Miguel de Tucumán: [s.n.], 1974.

51 EPIMÉLEIA. Buenos Aires: Universidad Argentina John F. Kennedy-Departamento de FilosofíaCentro de Investigaciones en Filosofía e Historia de las Religiones, $n^{\circ}$ 1: $1992-2009$.

52 EPISTEME. Teoría de las ciencias. Buenos Aires: Asociación Argentina de Epistemología, $\mathrm{n}^{\circ}$ : noviembre 1947 - vol. II, $n^{\circ}$ 3: 1971 . Directores: Luciano Allende Lezama y Armando Asti Vera. Entre 1970 y 1971, sus directoras fueron: Celia Pérès de Cambiaso, Magdalena Bleyle.

53 ESCRITOS DE FILOSOFíA. Buenos Aires: Academia Nacional de Ciencias-Centro de Estudios Filosóficos, $\mathrm{n}^{\circ}{ }^{1:} 1978-\mathrm{n}^{\circ} 43: 2003$. Dir.: Eugenio Pucciarelli.

54 LA ESCUELA POSITIVA. Corrientes: [s.n.], $\mathrm{n}^{\circ}$ 1: febrero de 1895-1896. Realizada por: Alfredo Ferreira, Ángel Scalabrini, Ángel C. Bassi, Víctor Mercante, Máximo S. Victoria, Modesto Leites.

55 ESPACIO. Órgano de la Asociación Universitaria de Estudiantes de la Facultad de Filosofía y Letras. Buenos Aires: UBA-FFyL, $n^{\circ}$ 1: 1957 - $n^{\circ}$ 10/11: julio/agosto 1958. Comité de redacción: José Alzola Zárate, María del Pilar Cámara, Ana María Cambours Ocampo, Julio Crespo, Mario Nascimbene, Beatriz Piemonte, Virginia Slarsky.

56 ESPACIOS de crítica y producción. Buenos Aires: UBA-FFyL, $n^{\circ} 1$ (1984) - [Publicación abierta]. Comité editorial: M. Boivin, C. Gorlier, J.E. Dotti, G. Palau A. Pucciarelli, J. Sazbón, P. Gentilli, J.E. Burucúa, A. Cattaruzza, M. Kohan.

57 ESTUDIOS. Buenos Aires: Universidad del Salvador -Academia Literaria del Plata, 1920-1970.
58 ESTUDIOS. Filosofía práctica e historia de las ideas. Mendoza:: INCIHUSA - CRICYT. 2000, no 1: diciembre 2000 - [publicación abierta]. Primer director.: Arturo Andrés Roig.

59 ESTUDIOS DE EPISTEMOLOGÍA. Tucumán: Universidad Nacional de Tucumán-Facultad de Filosofía y Letras- Instituto de Epistemología, $n^{\circ} 1$ : 1989 - [publicación abierta].

60 ESTUDIOS DE FILOSOFíA. Centro de Estudios de Filosofía y Humanidades. Córdoba: UNC, 1942[1943].

61 ESTUDIOS DE FILOSOFÍA Y RELIGIONES DE ORIENTE. Buenos Aires: UBA-FFyL-Centro de Estudios de Filosofía Oriental, nº 1971.

62 ESTUDIOS TEOLÓGICOS Y FILOSÓFICOS. Buenos Aires: Padres Dominicos Argentinos, $\mathrm{n}^{\circ} 1$ : 1959-vol III, $\mathrm{n}^{\circ} 3$ : 1961. Fueron sus directores: Dir.: Rubén C. González y Alberto García Vieyra. Secretario de redacción: Domingo Renaudiére de Paulis.

63 ETHOS. Revista de filosofía práctica. Instituto de Filosofía Práctica Dr. Guido Soaje Ramos, ${ }^{\circ} 1$ : 1973 - 1974-97: n²3/25.

64 FACUNDO. Crítica y polémica. Córdoba: [s.n.], 1935-1939. ํㅜ 1: mayo 1935 - no 7: diciembre 1939. Dir.: Saúl Taborda.

65 FILO. Revista del Centro de Estudiantes de Filosofía y Letras. Buenos Aires: UBA-CeFyL, $\mathrm{n}^{\circ} 1$ : noviembre 1983 - no 2: junio 1984. Dir.: Alejandro Cassini.

66 FILOSOFAR CRISTIANO. Córdoba: Asociación Latinoamericana de Filósofos Católicos, $n^{\circ} 1$ : 1977[1989]. Directores: Stanislav Ladusans, Alberto Caturelli y Agustín Basave.

67 FILOSOFÍA Y LETRAS. Órgano de los diplomados y alumnos de la Facultad de Filosofía y Letras. Buenos Aires: diplomados y alumnos de la Facultad de Filosofía, $n^{\circ}$ 1: noviembre $1901-n^{\circ}$ 9/10: agosto 1902. Comisión redactora: René Bastianini, Ricardo E. Cranwell, Celestina F. de Frutos, Jorge L. Grimmé, Eugenio Ivancovich, Andrés Mazzeo, Francisca R. de Páez, Augusto Rivas, Porfirio Rodríguez, Casimiro Toranzo Calderón.

68 HELIOS. Revista mensual. Literatura, Historia, Filosofía, Crítica, Pedagogía, Arte. Buenos Aires: Helios, $n^{\circ}$ 1: julio 1918 - nº 5 : noviembre 1918. Dir.: M. Conde Montero.

69 HIEROS. Revista de filosofía y religión. Buenos Aires: UBA-FFyL, $n^{\circ}$ 1: 1964. Dir.: Victor Massuh. 
70 HIPARQUIA. Revista de feminismo filosófico. Buenos Aires: Asociación Argentina de Mujeres en Filosofía (AAMEF), n 1: 1988 - n 10: 1999. Primer consejo de redacción: Ana María Bach, María Luisa Femenías, Alicia Gianella, Clara Kuschnir, Diana Maffía, Margarita Roulet y María Isabel Santa Cruz.

71 HUMANIDADES. Publicación de la Facultad de Ciencias de la Educación. La Plata: UNLP, $n^{\circ} 1$ : 1921- $n^{\circ}$ 38: 1961. Primer director: Ricardo Levene.

72 HUMANITAS. Revista de la Facultad de Filosofía y Letras. San Miguel de Tucumán: Universidad Nacional de Tucumán, no 1 (1953) - nº 24 (1977). Dir.: Diego Pro. Secretario: Manuel Gonzalo Casas.

73 IDEAS. Órgano de la Sección Estudiantes Universitarios del Ateneo Hispano Americano. Buenos Aires: Ateneo Hispano Americano, n-1: septiembre 1915 - n²1: marzo de 1919. Dir.: José M. Monner Sans, luego A. Britos Muñoz luego Francisco de Aparicio.

74 INICIAL. Revista de la nueva generación. Buenos Aires: Inicial, $n \div 1$ (octubre 1923) - n 11 (febrero 1926). Redactores: Roberto Ortelli, Brandán Caraffa, Roberto Smith, Homero Guglielmini.

75 INSTANTES Y AZARES. Escrituras nietzscheanas Buenos Aires: [s.n.], n¹: 2001 - [publicación abierta]. Dir.: Mónica Cragnolini. Continúa a: Perspectivas Nietzscheanas $\rightarrow$.

76 KAIRÓS. Revista de cultura y crítica estética. Buenos Aires: [s.n.], nํㅜㄹ: agosto 1967 - nำ 7 : septiembre 1969.

77 LIBERTAD CREADORA. Revista trimestral. La Plata: Claridad, no 1: enero/marzo 1943 - nㅇ 2: abril/junio 1943. Dir.: G. Korn Villafañe.

78 LOGOS. Revista de la Facultad de Filosofía y Letras. Buenos Aires: Universidad de Buenos Aires, $\mathrm{n}^{\circ} 1$ (primer trimestre 1941) - $\mathrm{n}^{\circ} 16$ (1980/1981). Dir.: Coriolano Alberini, luego Ángel Battistessa, luego Delfín Leocadio Garasa. Comisión consultiva: Coriolano Alberini, Rafael Alberto Arrieta, Alberto Freixas.

79 MÉTHEXIS. Revista argentina de filosofía antigua. Buenos Aires: UBA-FFyL- Instituto de Filosofía - Sección de Estudios de Filosofía Antigua, no 1 (1988) - [publicación abierta]. Director fundador: Conrado Eggers Lan. A partir de 1999 cambió su subtítulo a "International Journal for Ancient Philosophy” y comenzó a ser editada desde Holanda y Alemania.

80 MINERVA. Revista continental de filosofía. Buenos Aires: [s.n.], nº 1: mayo/junio 1944 - nº 5/6: enero/abril 1945. Dir.: Mario Bunge.
81 NOMBRES. Revista de filosofía. Córdoba: Universidad Nacional de Córdoba-Facultad de Filosofía y Humanidades. Área de FilosofíaCentro de Investigaciones., n- 1 (dic. 1991) - nº 28 (nov. 2014). Dir.: Gustavo Casacov, Carlos Longhini, Sergio Sánchez, Carolina Scotto, Diego Tatian, Oscar del Barco y José Moneo.

82 NOSOTROS. Revista mensual de letras, arte, historia, filosofía y ciencias sociales. Buenos Aires: Nosotros, $\mathrm{n}^{\circ} 1$ (agosto 1907) - $\mathrm{n} \div 300$ (diciembre 1934); segunda época, $n \cong 1$ (abril 1936) - no 93 (diciembre 1943). Dir.: Afredo A. Bianchi y Roberto F. Giusti.

83 NOTAS DE FILOSOFÍA DEL DERECHO. Buenos Aires: [s.n.], n¹: 1964 - n 5: 1969. Dir.: García Maynes, Jorge Bacqué, Eugenio Bulygin, Genaro Carrió, León Dujovne, Ambosio Gioja, E. Garzón Valdés.

84 NOTAS Y ESTUDIOS DE FILOSOFÍA. Tucumán: [s.n.], no 1: 1949 - n 17: 1954. Jefe de redacción: Juan Adolfo Vázquez.

85 ORIENTE-OCCIDENTE. Instituto Latinoamericano de Investigaciones Comparadas sobre Oriente y Occidente. Buenos Aires: Universidad del Salvador - CONICET, $\mathrm{n}^{\circ}$ 1: enerojun. 1980 - año 16, n 1/2 (1999).

86 PATRISTICA ET MEDIEVALIA. Buenos Aires: UBA-FFyL-Sección de Estudios de Filosofía Medieval, n¹: 1975 - [publicación abierta]. Dir.: Francisco Bertelloni. Secretaria de Redacción: Silvia Magnavacca. Prosecretaria: Claudia D’Amico.

87 EL PENSADERO. Revista de filosofía. Buenos Aires, [s.n.], nº1: julio $2005-\mathrm{n}^{\circ}$ 3: septiembre 2006. Dir.: María Jimena Solé. Consejo de redacción: D. Baranzelli, P. Cesario, M. Córdoba, G. Fernández Walker, L. Galazzi, J. Mayoral. M. J. Solé, E. Vergalito.

88 PENSAMIENTO DE LOS CONFINES. Buenos Aires: Fondo de Cultura Económica, ${ }^{\circ}{ }^{\circledR}$ : 1995 - n²8/29: 2012. Dir.: N. Casullo. Comité de redacción: R. Forster, M. Bruera, A. Kaufman.

89 PERSPECTIVAS NIETZSCHEANAS. Buenos Aires: [s.n.], n 1: 1992 - n8: 1998. Dir.: Mónica Cragnolini. Continúa como Instantes y azares $\rightarrow$.

90 PHILOSOPHIA. Anuario de filosofía. Mendoza: Universidad Nacional de Cuyo-Facultad de Filosofía y Letras- Instituto de Filosofía, $n^{\circ}$ 1: 1944 - n 46/47: 1987. Dir.: Ángel González Álvarez.

91 POLÍTICAS DE LA MEMORIA. Buenos Aires: Centro de Documentación e Investigación de la Cultura de Izquierda, $\mathrm{n}^{\circ}{ }_{1}$ : 1998 - [publicación abierta]. 
92 EL POSITIVISMO. Buenos Aires: Órgano del Comité Positivista Argentino, $\mathrm{n}^{\circ}$ 1: junio 1925 - $\mathrm{n}^{\circ}$ 76/77: julio-agosto 1938.

93 PRISMAS. Revista de historia intelectual. Bernal: UNQ - Programa de Historia intelectual, $\mathrm{n}^{\circ}$ 1: 1997 - [publicación abierta]. Consejo de Dirección: Carlos Altamirano, Elías Palti, Adrián Gorelik, Jorge Myers, Oscar Terán.

94 PUNTO DE VISTA. Revista de cultura. Buenos Aires: Punto de vista, $n^{\circ}$ 1: marzo $1978-n^{\circ} 90$ : abril 2008. Dir: Beatriz Sarlo.

95 REALIDAD. Revista de ideas. Buenos Aires: [s.n.], no 1 (enero/febrero 1947) - no 17/18 (septiembre/ diciembre 1949). Dir.: Francisco Romero.

96 REVISTA ARGENTINA DE CIENCIAS POLÍTICAS. Buenos Aires: [s.n.], 1910-1928. Dir.: Rodolfo Rivarola.

97 REVISTA ARGENTINA DE FILOSOFÍA y POLITICA. Buenos Aires: [s.n.], no 1: agosto 1995. Dir.: Pablo Anzaldi.

98 REVISTA DE FILOSOFÍA. La Plata: Universidad Nacional de La Plata.-Facultad de Humanidades y Ciencias de la Educación- Departamento de Filosofía, $n^{\circ} 1$ (1950) - $n^{\circ} 24$ (1982); nueva época: $n^{\circ} 25$ (1985) - [publicación abierta]. Dir.: Octavio N. Derisi, Emilio Estiú (segunda época). Denominada luego Revista de Filosofía y Teoría Política. Primer director: Octavio N. Derisi.

99 REVISTA DE FILOSOFíA. Asociación de Estudios Filosóficos. Buenos Aires: ADEF, vol I, $n^{\circ} 1$ : 1986vol. XVI, $n^{\circ} 1$ : 2001. Primer director: Abel Lasalle Casanave. Comité de redacción inicial: C. Amor, B. von Bilderling, M. Boeri, A. Cassini, P. García, C. Jáuregui, J. Legris, F. Schuster, A. Vigo.

100 REVISTA DE FILOSOFíA, CULTURA, CIENCIAS y EDUCACIÓN. Buenos Aires: [s.n.] no 1: enero 1915 - no 90: diciembre 1929. Dir.: José Ingenieros; entre 1923 y 1925 comparte la dirección con Aníbal Ponce; desde enero 1925: Aníbal Ponce.

101 REVISTA DE FILOSOFÍA JURÍDICA Y SOCIAL. Rosario: Universidad de Rosario -Facultad de Derecho-Centro de Investigaciones de Filosofía Jurídica y Filosofía Social, $n^{\circ}$ 1: 1996 [publicación abierta].

102 REVISTA DE FILOSOFÍA LATINOAMERICANA. Liberación y cultura. Buenos Aires: Asociación de Filosofía Latinoamericana y Ciencias Sociales, [primera época]: nº 1 (enero/junio 1975) - no 9/10 (enero/diciembre 1979); segunda época, $\mathrm{n}^{\circ} 11$ (1985) - no 22 (2000). Editor: Juan Alberto Cortés. Consejo de redacción: Osvaldo Ardiles, Mario Casalla, Enrique Dussel, Aníbal Fornari y Juan Scannone.
103 REVISTA DE HISTORIA DE LAS IDEAS. San Miguel de Tucumán: Universidad Nacional de Tucumán, no 1 (1950). Dir.: Roger Labrousse. Asesores: Emilio Carilla, Juan Adolfo Vázquez. Secretaria: María Elena Vela.

104 REVISTA DE HUMANIDADES. Publicación universitaria. Córdoba: Facultad de Filosofía y Humanidades de la Universidad Nacional de Córdoba, nº ${ }^{\circ}$ : marzo 1958 - nº 13/13: julio 1974. Dir.: Emilio Sosa López.

105 REVISTA DE LA FACULTAD DE FILOSOFÍA Y HUMANIDADES. Córdoba: UNC, 1949-1954. Continúa como Revista de Humanidades: publicación universitaria $\rightarrow$.

106 REVISTA DE LA SOCIEDAD ARGENTINA DE FILOSOFÍA. Córdoba: Sociedad Argentina de Filosofía, no ${ }^{\circ}$ : noviembre $1981-n^{\circ} 7$ : 1992.Dir.: Arturo García Astrada.

107 REVISTA DE LA UNIVERSIDAD NACIONAL DE CóRDOBA. Córdoba: UNC, nº 1 (ago. 1914) - 1972. Directores: Enrique Martínez Paz, Félix Garzón Maceda, Gregorio Berman, Ernesto Gavier, Augusto Schmiedecke, Sebastián Soler, Alfredo Fragueiro, Carlos E. Melo, Alfredo Poviña, Néstor A. Pizarro, Oscar E. Cocca, Ezio Masoni, Victor Enrique Sappia, Francisco Junyet Velez, Francisco Pereyra.

108 REVISTA DE LA UNIVERSIDAD DE BUENOS AIRES. Buenos Aires: UBA, [primera época:] nº 1 : marzo 1904 - no 158 : diciembre 1923 (Dir.: Rodolfo Rivarola; Mario A. Rivarola; Coriolano Alberini); segunda época: abril 1924 - noviembre 1931. Tercera época: julio 1933 - diciembre 1946 (Dir.: Emilio Ravignani); cuarta época: enero 1947-diciembre 1953 (Dir.: Hernán Benítez); Quinta época: enero 1956- diciembre 1963 (Dir.: Marcos Victoria y desde julio de 1960 por José Luís Romero).

109 REVISTA DEL CENTRO UNIVERSITARIO DE LA PLATA. La Plata: Centro Universitario de La Plata, $n^{\circ} 1$ : jul. 1900-Dir.: Vicente Añón Suárez.

110 REVISTA DEL INSTITUTO DE FILOSOFÍA. Córdoba: UNC, $\mathrm{n}^{\circ}$ 1: 1957 - 1958. Dir.: Juan Adolfo Vázquez.

\section{REVISTA LATINOAMERICANA DE FILOSOFÍA.}

Buenos Aires: Centro de Investigaciones Filosóficas (CIF), no 1: marzo 1975 - [Publicación abierta]. Comité inicial de redacción: Rafael Braun, Osvaldo Guariglia, Ezequiel de Olaso, Mario Presas, Eduardo Rabossi.

112 EL Río SIN ORILLAS. Revista de filosofía, cultura y política. Buenos Aires: Las Cuarenta, ${ }^{\circ}{ }^{\circ}$ :. 2007 - [nº: 2013]. Comité de redacción inicial: Diego Caramés, Mariana Casullo, Gabriel D’lorio, Matías Farías, Julián Fava, Guadalupe Lucero, Virginia Osuna, Mariana Santángelo. 
113 EL RODABALLO. Revista de política y cultura. Buenos Aires: El Cielo por Asalto, ํㅜ 1 (noviembre 1994) - no 16 (verano 2006). Consejo editor inicial: Raquel Ángel, Blas de Santos, Eduardo Grüner, Alberto Guilis, Horacio Tarcus.

114 SAGITARIO. Revista de humanidades. La Plata: [s.n.], no 1: mayo/junio 1925 - no 10/12: noviembre/ diciembre 1927. Dir.: Carlos A. Amaya, Julio V. González, Carlos Sánchez Viamonte.

115 SAPIENTIA. Revista tomista de filosofía. La Plata -Buenos Aires: Pontificia Universidad Católica Argentina, $n^{\circ}$ 1: 1946 - [publicación abierta]. Directores: Octavio N. Derisi y Guillermo Blanco.

116 SEMIRRECTA. Filosofía, literatura y arte. Buenos Aires: [s.n.], $n^{\circ} 1$ : agosto-septiembre $1952-n^{\circ} 6 / 7$ : 1953, Director: Corrado Eggers Lan. Subdirectora: Eugenia Marenco. Consejeros: Adolfo P. Carpio, Marcelo Nuñez Achard, Alfredo Vilariño Ochoa, Edgar Ruffo y Federico Borghini.

117 SEXTO CONTINENTE. Revista de cultura para América Latina. Buenos Aires: [s.n.], no 1: julio 1949 - n 7/8: noviembre/diciembre 1950. Dir.: Armando Cascella.

118 SÍNTESIS. Artes, ciencias y letras. Buenos Aires: [s.n.], no 1: junio 1927 - no 41: octubre 1930. Dir.: Xavier Bóveda, luego Martín S. Noel. Consejo asesor: Coriolano Alberini, J. Rey Pastor, Emilio Ravignani, Carlos Ibarguren, Martín S. Noel, Arturo Capdevilla, Jorge Luís Borges.

119 SOL Y LUNA. Buenos Aires: Sol y Luna, $n^{\circ} 1$ : 26/11/1938 - no 10: 28/5/1943. Dir.: Mario Amadeo, Ignacio B. Anzoátegui, Juan Carlos Goyeneche; Secretario de redacción: José María de Estrada.

120 SUSTANCIA. Revista de cultura superior. Tucumán: Sociedad Sarmiento, № 1: junio 1939 - no 18: noviembre/diciembre 1946. Dir.: Alfredo Coviello.
121 TAREA. Revista de iniciación filosófica. La Plata: UNLP-Departamento de Filosofía, 1968-1972.

122 TEOLOGÍA DE LA LIBERACIÓN. Buenos Aires: Servicio de Paz y Justicia, n ${ }^{\circ}$ : abril 1986.

123 TÓPICOS. Santa Fe: Universidad Católica de Santa Fe -Instituto de Filosofía, n 1: $1993-$ [publicación abierta].

124 UNIVERSIDAD. Santa Fe: Universidad Nacional del Litoral, 1935-1964. Dir.: Josué Gollán (h.) y Domingo Buonocore.

125 VALORACIONES. Humanidades, crítica y polémica. La Plata: Renovación, ํo-1 (septiembre 1923) - no 12 (mayo 1928). Dir.: Carlos Américo Amaya; desde el ${ }^{\circ} 6$, Alejandro Korn.

126 VERBUM. Revista del Centro de Estudiantes de Filosofía y Letras de la Universidad de Buenos Aires. Buenos Aires: Centro de Estudiantes de la Facultad de Filosofía y Letras, 끄 20 (1912) - no 90 (1948). Dir.: Carmelo M. Bonet; luego, sucesivamente: Carlos Bogliolo, Juan Probst, Jorge Zamudio Silva, Eduardo Vaccaro, Ángel Battistessa, Carlos A. Fayard; Regina Gibaja. Continúa al Boletín del Centro de Estudiantes de Filosofía y Letras $\rightarrow$.

127 VIDA Y ÉTICA. Buenos Aires: Pontificia Universidad Católica Argentina-Instituto de Ética Biomédica, $n^{\circ}$ 1: 2000 - [publicación abierta].

128 STROMATA. Buenos Aires: Universidad Nacional del Salvador - Facultad de Filosofía y Teología, $n^{\circ}$ 1: 1938 - [publicación abierta]. Fueron directores de esta publicación Enrique B. Pita, M. A. Fiorito y Ricardo Daniel Miño.

129 XENIUM. revista de filosofía. Córdoba: [s.n.], 1957-1959. Dir.: Alberto Caturelli 


\section{Referencias Bibliográficas}

" Alberini, Coriolano. 1966. Problemas de la Historia de las Ideas Filosóficas en la Argentina. La Plata: UNLP.

》Alberini, Coriolano. 1981. Precisiones sobre la evolución del pensamiento argentino. Buenos Aires: Docencia - CINAE.

»Altamirano, Carlos y Beatriz Sarlo. 2004. Entre el naturalismo y la psicología: el comienzo de la 'ciencia social' en la Argentina. En Neiburg, F. y M. Plotkin, comps. Intelectuales y expertos: la constitución del conocimiento social en Argentina. Buenos Aires: Paidós.

»Auza, Néstor. 1968. Estudio e índice general de la Revista Nacional (1886-1908). Buenos Aires: Universidad del Salvador.

»Auza, Néstor. 1980. Correo del domingo (1864-1868) (1879-1880): Estudio e índice general. Buenos Aires: Instituto Histórico de la Organización Nacional.

"Biagini, Hugo. 1985. Panorama filosófico argentino. Buenos Aires: Eudeba.

"Biagini, Hugo; Elena Ardissone y Raúl Sassi. 1979. Revista de Filosofía (19151929): Estudio e índices analíticos. Buenos Aires: Centro de Estudios Filosóficos - Editorial Docencia.

»Blanco, Alejandro. 2006a. Gino Germani: la renovación intelectual de la sociología. Bernal: UNQ.

»Blanco, Alejandro. 2006b. Razón y modernidad. Gino Germani y la sociología en la Argentina. Buenos Aires: Siglo XXI.

»Bulygin, Eduardo. 1996. Carlos Alchourrón (1931-1996). En Análisis Filosófico. Vol. XVI, no. 2, 110-114.

》Bunge, Mario. 2014. Memorias entre dos mundos. Buenos Aires: Eudeba-Gedisa.

»Bustelo, Natalia. 2012. La juventud universitaria de Buenos Aires y su vínculo con las izquierdas en los inicios de la Reforma Universitaria (1914-1922). En Izquierdas. No 16, 1-30.

»Bustelo, Natalia. 2014. La construcción de la familia estudiantil de la Reforma Universitaria: El Ateneo de Estudiantes Universitarios (1914-1920) de Buenos Aires y sus publicaciones periódicas Ideas y Clarín. En Políticas de la Memoria. No. $14,63-78$.

»Bustelo, Natalia y Lucas Domínguez Rubio. 2016. El antipositivismo como respuesta a la crisis civilizatoria. El proyecto filosófico-político de Alejandro Korn. En Cuadernos del Sur-Filosofía. No. 45.

»Bustelo, Natalia y Lucas Domínguez Rubio. 2017. Radicalizar la Reforma Universitaria. La fracción revolucionaria del movimiento estudiantil argentino, 19181922. En Anuario Colombiano de Historia Social y de la Cultura. Vol. 44, 31 - 62.

"Cassini, Alejandro. 1998. Los caminos hacia la profesionalización de la filosofía: las revistas argentinas de filosofía en el último cuarto de siglo. En Cuadernos de Filosofía. No. 43, 103-113.

»Cassini, Alejandro. 2017. La fragmentación de la filosofía: sus causas y sus consecuencias. En Revista Latinoamericana de Filosofía. Vol. 43, 105- 133. 
»Cattaruzza, Alejandro y Alejandro Eujanian. 2003. Políticas de la historia. Argentina 1860-1960. Madrid-Buenos Aires: Alianza.

"Caturelli, Alberto. 2001. Historia de la filosofía en la Argentina. Buenos Aires: Ciudad Argentina - UNSAL.

"Colegio Libre de Estudios Superiores. CLES. 1931-1952. Cursos y conferencias: Revista del Colegio Libre de Estudios Superiores. Índice julio 1931 a marzo 1952. Buenos Aires: Colegio Libre de Estudios Superiores.

»Delgado, Verónica. 2006. El nacimiento de la literatura argentina en las revistas literarias: 1896-1913. La Plata: UNLP.

»Delgado, Verónica. 2014. Algunas cuestiones críticas y metodológicas en relación con el estudio de revistas. En Delgado, Verónica; Alejandra Mailhe y Geraldine Rogers. Tramas impresas: Publicaciones periódicas argentinas (XIX-XX). La Plata: UNLP.

»Devoto, Fernando y Nora Pagano. 2009. Historia de la historiografía argentina. Buenos Aires: Sudamericana.

»Domínguez Rubio, Lucas. 2017. Filosofía e historia en las primeras historias de las ideas argentinas: la discusión historiográfica entre José Ingenieros y Alejandro Korn. En Prismas: revista de historia intelectual. No. 21, 75-94.

»Dotti, Jorge. 1992. La letra gótica: recepción de Kant en Argentina. Buenos Aires: Facultad de Filosofía y Letras.

»Ehrlicher, Hanno. 2014. El estudio de revistas culturales en la era de las humanidades digitales: Reflexiones metodológicas para un debate. En Delgado, Verónica; Alejandra Mailhe y Geraldine Rogers. Tramas impresas: Publicaciones periódicas argentinas (XIX-XX). La Plata: UNLP. p. 26-45.

"Eujanian, Alejandro. 2001. El novecentismo argentino: reformismo y decadentismo. La revista Cuaderno del Colegio Novecentista, 1917-1919. En Estudios Sociales. Vol. XI, no. 21, 83-105.

"Farré, Luis. 1958. Cincuenta años de filosofía argentina. Buenos Aires: Peuser.

» Fernández Vega, José. 2011. Lugar a dudas: Cultura y política en la Argentina. Buenos Aires: Las Cuarenta.

" Guedon, Jean-Claude. 2001. In Oldenburg's Long Shadow: Librarians, Research Scientists, Publishers, and the Control of Scientific Publishing. Washington: Association of Research Librarie.

» Gutiérrez, José María. 1998 [1868]. Noticias históricas sobre el origen y desarrollo de la enseñanza pública superior en Buenos Aires. Bernal: UNQUI.

» INIBI. Instituto de Investigaciones Bibliotecológicas. 200o. Índice de las Publicaciones de la Facultad de Filosofía y Letras [CD ROM]. Buenos Aires: INIBI-Facultad de Filosofía y Letras, Instituto de Investigaciones Bibliotecológicas-Universidad de Buenos Aires.

"Leocata, Francisco. 1993. Las ideas filosóficas en la Argentina. Buenos Aires: CESBA.

» Maeder, Ernesto. 1961. Índice general de la Revista de Buenos Aires (1863-1871). Resistencia: Universidad Nacional del Nordeste.

» Maeder, Ernesto. 1965. Índice general de la Revista de Buenos Aires (1871-1877). Resistencia: Universidad Nacional del Nordeste.

» Maeder, Ernesto. 1969. Los Anales de la Sociedad Científica Argentina: 1876-1930: descripción e índices. Resistencia: Universidad Nacional del Nordeste. 
» Maffia, Diana. 2010. El análisis filosófico y la universidad de las catacumbas. En Actas XV Congreso Nacional de Filosofía. Buenos Aires: AFRA.

"Masiello, Francine. 1986. Lenguaje e ideología: las escuelas argentinas de vanguardia. Buenos Aires: Hachette.

»Palau, Gladys. 1994. Cuadernos de filosofía y coyuntura. En Cuadernos de Filosofía. No. 40, 39-44.

"Patiño, Roxana. 2008. Revistas literarias y culturales. En Amícola, José y José Luis de Diego, dirs. La teoría literaria hoy. Conceptos, enfoques, debates. La Plata: Al Margen.

»Pluet-Despatin, Jacqueline. 1999. Une contribution à l'histoire des intellectuels: les revues. En Les Cahiers de L' IHTP. No. 20, 125-136.

»Prislei, Leticia. 2015. La primera década de Nosotros. En Prislei, Leticia. Polémicas intelectuales, debates políticos. Buenos Aires: EFFL. p. 27-47.

"Pro, Diego. 1973. Historia del pensamiento filosófico argentino. Mendoza: UNC.

»Rabossi, Eduardo. 1981. El análisis filosófico en Argentina. En Gracia, J.; E. Rabossi; E. Villanueva y M. Dascal, comps. El análisis filosófico en América Latina. México: FCE.

»Ramaglia, Dante. 2010. Condiciones y límites del proceso de institucionalización de la cultura filosófica argentina a comienzos del siglo XX. En Solar. No. 6, 13-29.

»Rodríguez, Fernando. 2004. Inicial: El frente estético-ideológico de la nueva generación. En Inicial. Revista de la nueva generación (1923-1927). Bernal: Universidad Nacional de Quilmes. p. 7-43.

»Rodríguez, Fernando y Karina Vásquez. 2001. Gritos y susurros en el Jardín de Akademos: El movimiento estudiantil reformista en La Plata a través de sus revistas (1923-1927). En Intellèctus. No. 2.

"Roldán, Darío. 2006. Crear la democracia: la "Revista argentina de ciencias políticas" y el debate en torno de la República Verdadera. Buenos Aires: Fondo de Cultura Económica.

"Romanos de Tiratel, Susana. 2008. Revistas argentinas de Humanidades y Ciencias Sociales. Buenos Aires: INIBI.

»Rossi, Luis. 1999. Los proyectos intelectuales de José Ingenieros desde 1915 a 1925: la crisis del positivismo y la filosofía en la Argentina. En Revista de Filosofía, Cultura, Ciencias, Educación (1915-1929): prólogo y selección de textos por Luís Rossi. Bernal: Universidad Nacional de Quilmes. p. 13-62.

"Sarlo, Beatriz. 1992. Intelectuales y revistas: razones de una práctica. En Le discours culturel dans las revues latino-américaines (1940-1970). Cahiers du CRICALL. No. 9/10, 9-16,

» Tarcus, Horacio. 1997. Insurrexit: Revista universitaria (1920-1921). En Lote. No. $8,1-5$.

" Tarcus, Horacio. 2004. Revistas, intelectuales y formaciones culturales izquierdistas en la Argentina de los 20. En Revista Iberoamericana. No. 208-209, 749-772.

»Varki, Elizabeth. 2017. Where Review Goes Wrong. En ACM Communications. Vol. 6o, no. 3, 29-30.

»Velarde Cañazares, Marcelo. 2013. La joven vanguardia filosófica de la década de 1920. En Cuyo. Vol. 30, no. 1, 61-87. 
